\title{
Sub-Penny and Queue-Jumping
}

\author{
Sabrina Buti \\ Rotman School of Management, University of Toronto \\ Francesco Consonni \\ Bocconi University \\ Barbara Rindi \\ Bocconi University and IGIER \\ Ingrid M. Werner \\ Fisher College of Business, Ohio State University
}

November, 2013 


\section{ABSTRACT}

Sub-Penny Trading (SPT) is a form of dark trading that allows traders to undercut displayed liquidity. We distinguish between SPT that is queue jumping (QJ) and midcrossing (MID) and find that QJ is higher for NASDAQ than NYSE stocks. Consistently with Buti, Rindi, Wen and Werner (2013), QJ is positively related to depth and negatively related to stock price. We also find that QJ is associated with improved lit market quality, especially for large capitalization stocks. Sub-penny quotes are allowed for stocks priced below $\$ 1.00$, and we use this fact to show that the spread improves but depth deteriorates as the price of a stock crosses from above to below $(\$ 1.00)$. 


\section{Introduction}

The microstructure of financial markets during the last decade has been characterized by a growing importance of inter-market competition between lit and dark venues, and a significant increase in high frequency trading (HFT). These two elements have fostered the growth of a trading practice called sub-penny trading (SPT) which now involves approximately $10 \%$ of the U.S. consolidated equity volume and which is the objective of the investigation in this paper.

SPT takes place when traders undercut orders at the top of limit order books (LOBs) by a fraction of the tick size, which is the minimum price increment allowed by regulators on financial trading platforms. SPT may either take place "in house" when broker-dealers match orders internally, against other clients' orders or against their own inventory. ${ }^{1}$ Or it may take place on dark pools, trading platforms that do not publicly display price quotes. $^{2}$ Broker-dealers may use dark pools to internalize customers' orders at sub-penny increments, but they may also use dark pools to post limit orders at sub-penny prices. Also institutional traders with access to dark pools may use them to post orders that jump ahead of the orders sitting at the National Best Bid Offer (NBBO) on the lit market. This queue-jumping feature of SPT in dark pools is particularly important for traders pursuing algorithmic and HFT strategies. Fast trading includes smart order routers (SORs) which are programs largely used by both the buy and the sell side to search for the best quotes in regular exchanges as well as in dark pools of liquidity. Therefore the use of SORs guarantees that orders posted at sub-penny increments are

\footnotetext{
${ }^{1}$ Broker-dealers' internalization accounts for a substantial share of the U.S. consolidated equity volume according to the Securities and Exchange Commission's Concept Release on Equity Market Structure (SEC, 2010), being equal to $17.5 \%$ in 2009. According to the SEC Concept Release, in 2009 around 10 exchanges, 5 electronic communication networks, 32 dark pools, and over 200 broker-dealers were active in the U.S..

${ }^{2}$ More precisely, SPT can be observed in specific dark markets called Bank-Brokers or Internalization Pools. According to Rosenblatt Securities Inc.'s estimates for January 2013, Bank-Brokers Pools represent $7.09 \%$ of U.S. consolidated equity volume, and approximately $50 \%$ of total dark volume.
} 
actually executed if they offer the best price. This is why the rise of dark markets combined with the development of fast trading facilities has paved the way for the expansion of SPT.

Market participants can trade in sub-penny increments because of an exception established by Rule 612 of Regulation National Market System (Reg NMS). Rule 612 prohibits sub-penny quoting by banning traders from accepting, ranking or displaying orders or quotations in price increments smaller than a penny. At the same time, however, Rule 612 does not prohibit SPT. Specifically, SPT is allowed provided that it does not result from executions of visible quotations in sub-penny increments. This can occur for two reasons. First, Reg NMS exempts Alternative Trading Systems (ATSs) from pre-trade transparency as long as they execute less than $5 \%$ of the average aggregate daily volume in a particular stock. This implies that a broker may operate an ATS with no pre-trade transparency (a dark pool) where invisible quotations can be posted in sub-penny increments. Second, Rule 612 allows broker-dealers to internally execute non-displayed orders (typically retail orders) provided that this is done in compliance with their duty of best execution and so that orders are filled at prices that are better than the NBBO.

Rule 612 was introduced to limit the negative effects of the decimalization which took place in the U.S. in 2001. ${ }^{3}$ The rationale for the smaller tick size was to lower trading costs for investors. The unanticipated consequences were the possible effects on LOBs' depth and on brokers' profits. The U.S. Securities and Exchange Commission (SEC) recognized that very small tick sizes could have detrimental effects on the liquidity of LOBs. In particular, the regulator realized that if traders could undercut limit orders sitting on the book by an economically insignificant amount, it would potentially reduce the incentive for traders to post limit orders at the top of the LOB, and therefore could

\footnotetext{
${ }^{3}$ In the U.S. from 2001 the minimum price improvement was gradually reduced to 1 cent.
} 
have a detrimental effect on inside depth. The SEC also realized that a smaller tick size and hence a lower inside spread would lead to lower profit opportunities for brokers. Therefore, the SEC introduced an exception to the Rule so that broker-dealers could internalize small orders and at the same time offer their clients best execution by trading in sub-penny price increments inside the NBBO. As will be clarified later in this paper, another aspect of Rule 612 is relevant for our analysis of SPT. Rule 612 established a two-tiered market by setting a minimum price increment of $\$ 0.01$ (1 penny) only for stocks priced over $\$ 1$, and a minimum price increment of $\$ 0.0001$ for stocks priced below $\$ 1$.

For all the reasons discussed above SPT is one of the main concerns of the SEC and in this paper we aim at studying SPT in NASDAQ and NYSE stocks with the objective to understand its relevance, the factors which induce traders to undertake SPT and the effects that SPT may have on the quality of financial markets. We then tackle the regulatory issue of how to cope with the possible negative effects of SPT, and more generally how to choose the appropriate regulatory policy to influence SPT.

Our empirical analysis is based on a sample of Trade and Quote (TAQ) data for U.S. equities which includes all trades executed in sub-penny increments during 42 trading days in 2010. These trades may come from broker-dealers' internalization or from dark pools' executions. We build a stratified sample of 90 NASDAQ and 90 NYSE listed stocks and use Fama-MacBeth regressions to investigate which factors are associated with more SPT, i.e., under which conditions broker-dealers trade more intensively at sub-penny increments. We consider spread, depth, stock price, share volume, volatility and order imbalance. We also study in which type of stocks, small or large cap, low or high priced, there is more SPT as a fraction of consolidated volume. We then use a simultaneous equations model that takes into account endogeneity issues to investigate the association between SPT and market quality, measured by relative quoted spread 
and inside depth.

Before summarizing the main results of the paper, it is important to clarify how we classify SPT. We generally classify an execution as SPT when the price improvement associated with the execution price is smaller than $\$ 0.01$. However, note that there are two main reasons why we observe in the data trades executed on ATSs at fractions of a penny. First, traders may aim to undercut orders posted at the top of transparent LOBs. Second, the execution system of some dark pools follows a derivative pricing rule according to which all trades in the pool execute at the midpoint of the primary market's inside spread. ${ }^{4}$ To differentiate between these two categories, we treat executions at half a cent price improvement as a separate type of SPT and name it Mid-Crossing (MID), as it may originate from dark pools with midpoint derivative execution systems. ${ }^{5}$ We group the remaining SPT with a positive sub-penny price improvement different from half-cent into a category that we label Queue-Jumping (QJ). This way we adopt a parsimonious classification of QJ and we are sure that we do not mix data which derive from substantially different trading strategies and therefore could have different driving factors and different effects on the quality of the lit markets. However, by doing this we miss those executions originating from undercutting at exactly half a penny. ${ }^{6}$ In our study we will focus on QJ that is the main concern of regulators, and use MID primarily to highlight the differences with QJ.

To make predictions about the factors affecting QJ and about the effects of QJ on market quality, we rely on Buti, Rindi, Wen and Werner (BRWW, 2013) who model competition between a public LOB and a sub-penny venue (SPV). The model predicts that SPT takes place mainly for liquid low priced stocks and that the effects of SPT on

\footnotetext{
${ }^{4}$ To mention but a few, see ITG Posit, Liquidnet and BATS.

${ }^{5}$ While not included in our dataset, mid-quote executions can also occur within exchange operated dark pools.

${ }^{6}$ We also miss instances of QJ in stock at the whole penny increment, which may occur in high-priced and wide-spread stocks.
} 
market quality are negative for illiquid, especially low priced, stocks, and positive for liquid, especially high priced, stocks.

Our results are as follows. We find that for our sample QJ is on average $7.16 \%$ of the consolidated volume for NASDAQ stocks and 6.03\% for NYSE stocks. Consistently with the model's predictions, QJ is positively related to the liquidity of the stock, increasing with depth and decreasing with inside spread. QJ is also negatively related to the price of the stock; it is somewhat positively related to volatility and, for the group of large capitalization stocks, to the relative order imbalance. The opposite holds for small capitalization stocks for which QJ decreases with relative order imbalance. Still in line with the model's predictions, we find that for large capitalization stocks QJ positively affects bid depth and the inside spread, measured both in cents and in basis points, whereas we do not find significant results for the group of small stocks. By contrast, our results show that MID executions increase with share volume and order imbalance but have no significant effects on market quality, measured either by depth or inside spread.

Having investigated the overall magnitude and effects of QJ and MID, we extend the empirical analysis to address the regulatory issue. So far, two opposite proposals have been put forward from regulators and exchange officials that relate to SPT. In 2010, the SEC in its Concept Release on Equity Market Structure stressed that the larger percentage spread that characterizes low-priced stocks may lead to greater internalization by Over-The-Counter (OTC) market makers or more trading volume in dark pools, and proposed a reduction in the tick size for lower priced stocks.

While major U.S. exchanges, e.g., NYSE, NASDAQ and BATS, in their comment letters to the SEC concept release responded positively to the suggestion of reducing the tick size, there was at the same time a widespread sentiment among practitioners that decimalization curtailed brokers' profits and therefore incentives to supply liquidity for less liquid stocks and for initial public offerings (IPOs). This led to the 2012 Jumpstart 
Our Business Startups Act (Section 106(b) of the "JOBS Act") which instructed the SEC to investigate the possible effects of raising the minimum price increment, i.e., the tick size, for stocks of high growth companies and authorized the SEC to set a pilot program to raise the tick size for small and medium capitalization stocks to $\$ 0.10$. Our empirical work aims both at assessing whether an increase or a decrease in the tick size is the optimal regulatory reaction to the widespread use of SPT, and more generally whether the tick size is the right policy instrument for the regulator to use.

The SEC concept release and the following reactions of market players stress that it is the tick-to-price ratio that matters for policy. It is the size of the minimum price increment relative to the price of the stock that is the relevant policy instrument to investigate when dealing with QJ. ${ }^{7}$

Our results from the Fama-MacBeth regressions allow us to draw a first conclusion on the effect of a change in the relative tick size on QJ as we find a strong negative relation between QJ and the price of the stock. Because the sample of NASDAQ and NYSE stocks described above includes only stocks priced above $\$ 1$, the tick size is constant for that sample and therefore when the price increases the tick-to-price ratio decreases. This means that the negative relation between QJ and the stock price can also be interpreted as a positive relation between QJ and the tick-to price ratio. Therefore we can conclude that a reduction in the tick-to-price ratio leads to a reduction in QJ, and that if the aim of regulators is to reduce QJ, then the right policy action to take is to decrease rather than to increase the tick size.

\footnotetext{
${ }^{7}$ The SEC (2010) concept release (page 72) reads: There may be greater incentives for broker-dealer internalization in low-priced stocks than in higher priced stocks. In low-priced stocks, the minimum one cent per share pricing increment of Rule 612 of Regulation NMS is much larger on a percentage basis than it is in higher-priced stocks. For example, a one cent spread in a \$20 stock is 5 basis points, while a one cent spread in a $\$ 2$ stock is 50 basis points - 10 times as wide on a percentage basis. Does the larger percentage spread in low-price stocks lead to greater internalization by OTC market makers or more trading volume in dark pools? If so, why? Should the Commission consider reducing the minimum pricing increment in Rule 612 for lower priced stocks?
} 
An important caveat, however, is that within our sample all market participants willing to trade at sub-penny are constrained by Rule 612 to do it in the dark and we cannot therefore evaluate the counterfactual. We cannot investigate whether by liberalizing SPT, i.e., by reducing the tick size in regular exchanges, traders would actually move out of the dark and back into the lit markets.

To investigate this issue we exploit the fact that Rule 612 restricts SPT on lit markets only for stocks priced over $\$ 1$, while allowing trading in sub-penny increments for stocks priced below this threshold. We build a new sample of NASDAQ stocks whose price is between $\$ 0.8$ and $\$ 1.2$ at September 30, 2010 and which cross, at least once, the $\$ 1$ threshold during the sample period. We then study QJ around the regulatory threshold. Interestingly and contrary to our expectations, the proportion of SPT on ATSs for stocks priced below $\$ 1$ is greater than that for stocks priced above the threshold. This suggests that if SPT were allowed in the lit market for stocks priced above $\$ 1.00$, SPT on ATSs would not necessarily decrease.

Furthermore, to investigate the effects of QJ on market quality, we study the effects of SPT on spread and depth around the $\$ 1$ threshold by means of a discontinuity regression analysis. We find that for stocks priced below $\$ 1$, for which there is no constraint on SPT, more SPT is associated with a narrower spread but also with a deterioration of depth. This result suggests that a liberalization of SPT would potentially magnify some of the negative effects of QJ. However, we suspect that this result may be due to different make-take fee structures that could create strong incentives for traders to execute in the dark irrespective of Rule 612. Either way, we conclude that a change in the tick size does not seem to be the right policy action to take in order to reduce QJ or even to bring QJ back to lit venues.

The paper is organized as follows. In Section 2 we discuss the related literature and in Section 3 we lay out our testable hypotheses. In Section 4 we describe the dataset; in 
Section 5 we investigate the factors that affect SPT; in Section 6 we show the effects of SPT on market quality and in Section 7 we present the results from our discontinuity regression analysis. We conclude in Section 8.

\section{$2 \quad$ Related Literature}

As SPT takes place on dark venues, our paper is related to the empirical literature on dark pools. Ready (2013) investigates the determinants of dark trading by considering monthly volume by stock for the period June 2005 to September 2007 in two dark pools, Liquidnet and ITG POSIT, that executed approximately $1 \%$ of total market consolidated volume. He finds that dark pools execute most of their volume in stocks with low spread and high share volume. Considering that both Liquidnet and ITG POSIT execute at the mid-quote of the primary market spread, Ready's results are comparable (and consistent) with our findings for mid-crossing which show that the effect of depth on MID is positive and that of spread is negative.

Our results are also consistent with Buti, Rindi and Werner (BRW, 2011) who examine a unique dataset for the calendar year 2009 on dark pool activity for a large cross section of U.S. securities. BRW find that liquid stocks are those characterized by more intense dark pool activity. They also find that dark pool volumes increase for stocks with narrow quoted spreads and high inside bid depths, suggesting that a higher degree of competition in the LOB enhances dark pool activity. BRW also investigate the effect of dark trading on market quality and show that increased dark pool activity improves spreads, depth, and short-term volatility. Degryse, de Jong and van Kervel (2011) consider a sample of 52 Dutch stocks and analyze both internalized trades and trades sent to dark pools. They find that when these two sources of dark liquidity are

combined, the overall effect on global liquidity is detrimental. Hatheway, Kwan and 
Zheng (HKZ, 2013) too study the effects of dark trading on market quality, even though they only focus on effective spread. HKZ look at a sample of NASDAQ and NYSE stocks during the period January-March 2011 and find a positive relation between dark pool market share and effective spread. They explain this result with the effects of SPT that should drive uninformed traders away from the lit markets into dark pools thus increasing adverse selection costs on lit markets. We cannot directly compare HKZ' results with ours because they consider dark pool activity as a whole and do not distinguish between dark trading and SPT when looking at the effects on market quality. However, by controlling for endogeneity we find that SPT does not harm spread and depth at the top of the lit market which does not seem consistent with the conjectured cream skimming effect. Our paper is also related to Kwan, Masulis and Mc Inish (2013) who study the effects of competition for order flow on the fragmentation of U.S. equity markets and point out the relevance of SPT, and in particular of QJ, in the distribution of market shares across lit and dark venues. More precisely, they consider NASDAQ and NYSE stocks and show that when the price of a stock crosses the $\$ 1$ threshold, volume in dark venues increases while volume in traditional exchanges decreases.

Finally, Comerton-Forde and Putnis (2013) analyze the effect of dark trading on price discovery by considering the 500 largest stocks listed at the Australian Securities Exchange (ASX). They observe that informational efficiency and price discovery are negatively related to the share of volume executed on dark venues. However, they do not find any evidence that block trades harm price discovery. Still on price discovery, Nimalendran and Ray (2012) study detailed data from one dark pool and find evidence suggesting that price discovery may take place in the dark venue, particularly for less liquid stocks.

On the theory side, our paper is related to the models that study how limit order 
books work. ${ }^{8}$ As SPT crucially depends on the tick size and the state of the book -measured by depth and spread- to deliver testable empirical predictions the model must have discrete prices. Moreover, the model's equilibrium prices cannot be derived under the assumption of steady state which would inevitably imply a constant state of the LOB. ${ }^{9}$ To the best of our knowledge, the only theoretical model that satisfies these requirements and explicitly analyzes QJ is BRWW (2013). We refer to Section 3 for a discussion on this paper that we use to draw our main empirical predictions.

\section{Empirical Predictions}

$\mathrm{SPT}$ in the form of QJ is a recent practice that is attracting the attention of regulators, market officials and in general market participants. This paper aims to investigate whether QJ is detrimental or beneficial to the quality of the market, and to discuss the policy actions recently proposed. To this end it is important to understand the factors that may induce traders to engage in QJ.

We draw our main empirical predictions from BRWW (2013) which models competition between a LOB and a SPV. The SPV works like an opaque LOB but has a smaller tick size and hence a finer price grid than the public LOB. Traders access limit orders on the SPV through SORs which seek the best prices available in the two markets. Hence broker-dealers may have an incentive to step ahead of limit orders posted at the top of the public LOB by submitting limit orders on the SPV. BRWW (2013) shows that traders engage more intensively in QJ when competition for the provision of liquidity is strong on the LOB, i.e., when the LOB is deep and the spread is small. With greater inside depth the queue at the best bid and offer is longer, so that the incentive to gain

\footnotetext{
${ }^{8}$ To mention but a few, see Buti and Rindi (2013), Goettler, Parlour and Rajan (2005) and Parlour (1998).

${ }^{9}$ See, e.g., Foucault (1999), Foucault, Kadan and Kandel (2005) and Rosu (2009).
} 
price priority through QJ is greater. With smaller spread there is less room to post limit orders within the inside spread on the public LOB and therefore there is more incentive for traders to undercut existing liquidity via QJ on dark venues. ${ }^{10}$ This leads to our first prediction on the factors driving QJ.

Prediction \#1 QJ increases when the book is deep and when the inside spread is narrow.

BRWW (2013) also shows that the effect of the LOB liquidity on the propensity of traders to undercut via QJ is inversely related to the price of the stock. When the stock price is low, the costs of non-execution are small and therefore traders' propensity to supply rather than demand liquidity is high. It follows that competition for the provision of liquidity is higher for low priced stocks, and so is the incentive to undertake QJ in dark markets. This leads to our second prediction.

Prediction \#2 QJ is higher for low priced stocks.

QJ can take place in dark venues that allow executions in sub-penny increments, and trading in the dark can be affected by volatility and order imbalance. BRW (2011) show that for a given stock dark pool activity is higher on days with low intraday volatility and low order imbalances relative to share volume. In general traders approaching dark markets are worried about the uncertainty of executions and this uncertainty may increase with volatility, thus suggesting a negative relation between dark trading and both volatility and order imbalance.

Prediction \#3 QJ increases when both volatility and order imbalance decrease.

We now move to the predictions on the effects of QJ on the quality of regular exchanges. When traders move to dark venues to undercut the liquidity posted at the top of lit markets, not only liquidity supply but also liquidity demand moves away from

\footnotetext{
${ }^{10}$ For example, when the inside spread is equal to the tick size (very liquid stocks), traders cannot gain price priority on lit markets.
} 
regular exchanges, thanks to SORs that search for best execution in both lit and dark markets. So, the effects of QJ on the quality of regular exchanges depend on the relative proportion of limit and market orders that migrate from lit to dark venues. If the reduction in liquidity supply prevails, market quality -measured by spread and depthon lit markets decreases; if instead it is the reduction in liquidity demand that prevails, liquidity improves as the drop in market orders preserves liquidity on regular exchanges.

As shown in BRWW (2013), when the stock is liquid traders tend to use more market than limit orders and the second effect prevails. When instead the stock is illiquid, and there is no liquidity pressure at the top of the LOB, fewer and less aggressive limit orders are submitted to the LOB due to the perceived competition from QJ. Moreover, when the stock is illiquid traders tend to use more limit than market orders, so this time it is the first effect that prevails and liquidity worsens on the regular exchange. These results are summarized in the following prediction.

Prediction \#4 QJ improves (worsens) depth and spread when the stock is liquid (illiquid).

The policy debate on SPT aims at identifying the right policy instrument which regulators can use to influence QJ. BRWW (2013) shows that when the tick size decreases in a public LOB that competes with a SPV, SPT decreases as broker-dealers have lower incentives and profits from undercutting in the dark. This leads to our final empirical prediction.

Prediction \#5 A reduction in the tick-to-price ratio reduces QJ.

It is less straightforward to present predictions for MID because when MID is reported OTC, it could be either SPT or trading in dark pools which execute at the midpoint of the spread. Therefore we focus on the predictions for QJ. 


\section{Data Description}

\subsection{Data and Sample}

We construct a sample of stocks stratified by price and market capitalization for both NASDAQ and NYSE. As of December 31, 2009 we identify all common stocks in CRSP which are NYSE or NASDAQ listed. Then we divide all NYSE listed common stocks (i.e., share code 10 or 11), into terciles by market capitalization and price and form nine mutually exclusive groups with the same dimension. From each group we randomly draw 10 stocks for a total of 90 NYSE stocks. We repeat the procedure for NASDAQ stocks using the NYSE breakpoints. This way we create groups of stocks which are comparable across exchanges by market capitalization and price. Our final sample includes the 180 stocks reported in the Online Appendix (Tables A1-A2), and spans from October 1 to November 30, 2010, for a total of 42 trading days.

\subsection{Descriptive Statistics and Definitions}

The data we use come from the following sources. Data on number of shares outstanding, market capitalization, closing prices, listing exchanges and share codes are from CRSP. Data for S\&P 500 Stock Price Index (SP500) and CBOE S\&P 500 Volatility Index (VIX) used to capture market-wide activity are from Federal Reserve of Economic Data (FRED). Our main data source is the TAQ database which we describe in more detail below.

TAQ contains intraday transactions data (trades and quotes) for all securities listed

on the NYSE and American Stock Exchange (AMEX), as well as NASDAQ. The TAQ database includes a flag indicating the exchange where the trade was executed or the quote displayed. Each exchange is identified by a symbol/capital letter (except for 
NASDAQ which has two equivalent symbols, T and Q). The letter "D" is used for the Trade Reporting Facility (TRF) and for the NASD Alternative Display Facility (ADF). These facilities record transactions from OTC markets, some non-exchange ECNs, broker internalization and other dark pools. The crucial information for our analysis is that dark pools trades are reported as "D". The reason is that dark pools do not have to publicly report their quotes and so they do not have to comply with Rule 612 of Reg NMS, which only refers to quoted prices. As a result all sub-penny trades are reported with the exchange code " $\mathrm{D}$ ". ${ }^{11}$

We consider only trades and quotes which take place between 9:30:00 AM and 4:00:00 PM. For each day and for each stock we derive the NBBO using the Wharton Research Data Services (WRDS) suggested procedure and we compute the time-weighted bid, ask and total depth, the time-weighted quoted spread in cents and in percentage of the mid-quote, and the intraday price range, defined as (high-low)/high, as a measure of intraday volatility.

We remove erroneous and irregular trades; in particular we keep only trades whose correction indicator is either " 00 " or " 01 ". We then compute the share volume and (buy) order imbalance defined as the absolute value of (buys-sells)/share volume where buys are classified using a modified Lee and Ready (1991) algorithm. ${ }^{12}$ Table 1 shows descriptive statistics for our sample of stocks divided by exchange and capitalization.

[Insert Table 1 here]

\footnotetext{
${ }^{11}$ We can also observe sub-penny trades with other exchange codes. In this case, however, the price improvement is exactly equal to half-cent and is the result of a trade in exchange operated dark pools pricing at the NBBO mid-quote.

${ }^{12}$ We first apply a tick-test considering at most two previous trades. We classify a trade as a buy if its price is above the price of the previous trade (or two trades before); otherwise we classify the trade as a sell. If the trade is still unclassified, we classify it as a buy (sell) if the execution price is above (below) the mid-quote at the time of the trade.
} 


\subsection{Price Improvement}

In order to identify and classify SPT, we construct an auxiliary variable, the price improvement (PI), which is computed as follows. First, prices are rounded up to the closest cent for sell orders and rounded down to the closest cent for buy orders. Second, PI is obtained as the difference between the rounded price and the reported price. It is always restricted to the interval $(0.00,0.01)$. Therefore PI is equal to:

$$
P I=\mid \operatorname{rounded}(\text { price })-\text { price } \mid
$$

Table 2 reports an example of how we compute PI. We are now able to properly define SPT as a function of PI. In particular, we have no SPT when PI is equal to zero and SPT when PI is different from zero.

[Insert Table 2 here]

SPT can be further classified into MID, when PI is exactly equal to half-cent (0.005), and QJ, when PI is strictly positive but different from half-cent. ${ }^{13}$ Hence, our rounding procedure is immaterial to the definition of SPT, MID and QJ.

We can use PI to illustrate how significant SPT is in the U.S. equity markets. Interpreting PI as the gain from SPT and multiplying it by the number of shares traded in SPT, we obtain the dollar volume captured by stepping ahead of the queue. For example, consider Adobe Systems Inc.: in our sample period the daily average of shares traded is 14.8 million. Of these, 1.62 million are traded in SPT divided between QJ (1.25 million) and MID (0.37 million), which correspond respectively to $8.4 \%$ and $2.5 \%$

\footnotetext{
${ }^{13}$ Our measure of MID is a lower bound of executions at the mid-quote. The reason is that we are considering as mid-crossing only those executions which result in a sub-penny price. If the spread turns out to be an even multiple of the tick, we will not classify it as MID with our methodology.
} 
of consolidated share volume. Therefore the gross gain from SPT, measured as PI multiplied by the corresponding number of shares traded, is equal on average to $\$ 2,500$ for QJ and $\$ 1,850$ for MID on a daily base.

Figures 1 and 2 report the plot of the average QJ and MID across the sample period for each stock as a function of price, for NASDAQ and NYSE. Graphically we see that on both markets QJ generally decreases with price, while MID increases. Overall the percentage of volume traded in SPT increases when the stock value decreases and seems to be consistent with the fact that a one cent tick size is a binding constraint for low priced stocks. ${ }^{14}$

[Insert Figures 1-2 here]

To study the distribution of PIs, in Figures 3 - 4 we group stocks into 10 bis of size 0.001 for two groups of 30 high priced NASDAQ stocks and 30 high priced NYSE stocks, separately. For each bin, we report the associated SPT dollar volume as a percentage of the total traded volume; measuring it in share volume or number of trades yields qualitatively the same results. For example, Figure 3 shows that $3.05 \%$ of total NASDAQ traded volume is executed with a PI which lies in the interval $(0,0.001]$, while for NYSE it amounts to $2.44 \%$. The most common type of SPT corresponds to PI equal to 0.005. The distribution of PIs decreases almost monotonically as the PI increases, except for the spike at PI exactly equal to half-cent.

[Insert Figures 3-4 here]

\footnotetext{
${ }^{14}$ Tables "QJ Summary" and "MID Summary" in the Online Appendix show how QJ and MID are distributed between NASDAQ and NYSE across market cap and stock price.
} 


\section{$5 \quad$ Factors Driving Sub-Penny Trading}

To study how SPT varies with market characteristics, and test Predictions 1 and 2, we start with daily Fama-MacBeth cross-sectional regressions for both QJ and MID. More precisely, the regressors used in the different specifications are the following: dummy variable which is equal to one when the stock is NYSE listed, log of market capitalization, log of share volume, closing price, time-weighted cent quoted spread, time-weighted percent quoted spread, log of time-weighted bid depth, relative order imbalance in percent and intraday price range. The average daily estimated coefficients and t-statistics are reported in Tables 3 - 4. The t-statistics are based on the Newey-West adjusted standard errors with 5 lags.

[Insert Tables 3-4 here]

In specification (1) we control for listing exchange by including a dummy variable for NYSE listing. We also control for the logarithm of market capitalization. The results show that QJ is decreasing in market capitalization and is higher for NASDAQ than for NYSE stocks after controlling for market capitalization. Conversely, MID is increasing in market capitalization and is higher for NYSE than for NASDAQ stocks.

In specification (2) we replace market capitalization with share volume and closing price, and the results show that QJ is decreasing in price, while MID is increasing in price.

We then add the quoted spread in cents and the log of (time-weighted) bid depth in specification (3) to include measures of liquidity and we find that stocks with greater depth have more QJ and less MID. A wider quoted spread is also associated with higher MID, holding listing exchange, share volume, and price constant.

We replace quoted spread in cents and price with quoted spread in basis points in 
specification (4) and find that this variable is statistically significant only for QJ. Stocks with wider basis point spreads have more QJ, controlling for listing exchange and share volume. In addition, with this measure of spread the coefficient on bid depth is still statistically significant and positive.

Finally, in specification (5) we drop share volume and include the relative order imbalance in percent of share volume and volatility as measured by the intraday price range. We find that for the whole sample of NASDAQ and NYSE stocks the relation between QJ and volatility is not significant. However, by looking at the results for the different groups of stocks we find that while for small cap stocks the coefficient is still not significant, it is positive and significant for large capitalization stocks. We also find that MID increases significantly in relative order imbalance. ${ }^{15}$

In sum the multivariate Fama-MacBeth regression analysis shows that QJ is significantly higher for NASDAQ than NYSE stocks all else equal, while for MID we observe the opposite. In terms of our predictions, we find that QJ is positively related to time weighted bid depth and negatively related to price. These results are consistent with both Predictions 1 and 2. When the book is deep competition for the provision of liquidity is high and therefore QJ becomes a very attractive option for liquidity providers. Moreover the effect is stronger for low priced stocks as, when the price is low, the gain traders make by undercutting orders at the top of regular exchanges by a fraction of the tick size is higher as a percentage of the stock price.

The results for spread do not confirm the negative relation with QJ outlined in Prediction 1. The coefficient for quoted spread is not significant and that for percent spread is positive. This could be due to the interaction between spread and price. As discussed above, when the stock price decreases the costs of non-execution are smaller and the gains from QJ are higher so that traders are more inclined to use limit rather

\footnotetext{
${ }^{15}$ Results for different groups of stocks and for robustness checks are presented in the Online Appendix.
} 
than market orders. Hence submitting aggressive limit orders via QJ increases. However, when the price decreases, the relative spread increases with the result that the relation between relative spread and QJ becomes positive. Moreover, the spread itself can be influenced by QJ and therefore to find a clean relation between QJ and spread we need to control for endogeneity, which we do in the next Section.

Finally, our results on volatility and order imbalance do not confirm Prediction 3. This may be because a significant fraction of QJ is due to internalization for which some factors driving dark pool trading in separate venues may not be as relevant. Even though higher volatility increases execution uncertainty, if broker-dealers are active on both sides of the market, they might be insured against unexpected changes in the fundamental value of the asset.

\subsection{Robustness Checks}

As a first robustness check we rerun the Fama-MacBeth regressions separately for NASDAQ and NYSE listed stocks and we find that the results are qualitatively similar. We also rerun regressions for all the specifications by substituting the time-weighted bid depth with the time-weighted total depth and the results are unchanged. ${ }^{16}$ Second, we estimate the models separately for small and large capitalization stocks over the sample period, and we find that the positive relation between market capitalization and MID is due to small cap stocks. We also find that QJ is negatively related to relative order imbalance for small cap stocks and positively related for large stocks. This explains why overall the relative order imbalance is not significant.

\footnotetext{
${ }^{16}$ To economize space we do not report these results which are available upon request.
} 


\section{Sub-Penny Trading and Market Quality}

We now move to test our predictions on the effects of SPT, and in particular QJ, on the quality of regular exchanges. The issue is that market quality and dark trading are jointly determined as pointed out in BRW (2011), so to establish a causal relationship we have to address the endogeneity issue.

To deal with the inherent endogeneity of SPT and market quality, we need to find good instruments for SPT and market quality, respectively. In a recent paper studying the impact of low latency trading on market quality, Hasbrouck and Saar (2013) proposes using low latency trading in other stocks during the same time period as an instrument for low latency trading in a particular stock. We follow their suggestion and use SPT for other stocks (not i) on day t as an instrument for SPT in stock i. Because we have observed that there are systematic differences between exchanges and across market capitalization groups in SPT, we refine their instrument slightly. We require that the other stocks (not i) are listed on the same exchange as stock $\mathrm{i}$ and that their market

capitalization is in the same market capitalization group as stock i. The market quality measures are the time-weighted percent quoted spread and the logarithm time-weighted bid-depth. We estimate a two-equation simultaneous model for SPT, which can be either QJ or MID, and market quality measures (MQMs) using both traditional 2SLS and a two step generalized method of moments (GMM) procedure. Specifically, we estimate the following two-equation simultaneous model for each MQM:

$$
\begin{aligned}
M Q M_{i, t} & =a_{1} S P_{i, t}+a_{2} M Q M_{N O T i, t}+\varepsilon_{1, t} \\
S P_{i, t} & =b_{1} M Q M_{i, t}+b_{2} S P_{N O T i, t}+\varepsilon_{2, t}
\end{aligned}
$$

As instruments for $S P_{i, t}$, we use $S P_{N O T i, t}$ which is the average SPT of other stocks 
listed on the same exchange, in the same market capitalization group. Note that we exclude stock i. Similarly, as an instrument for $M Q M_{i, t}$, we use $M Q M_{N O T i, t}$, which is the average market quality measure for other stocks listed on the same exchange, in the same market capitalization group. We again exclude stock i. This estimation method is chosen to address the endogeneity of SPT and MQM. So we obtain a consistent estimate of the $a_{1}$ coefficient that tells us how SPT affects market quality.

We estimate the above system of equations for all stocks and days in a panel. To control for stock fixed effects, we de-mean all variables by deducting the in-sample average and divide the de-meaned variables by their in-sample standard deviation. As a result, the estimated coefficients can be interpreted as the response to a one standard deviation shock. We do not include any trend in the system nor we detrend our variables since the visual inspection of the data tells us that the variables are stationary in the sample period. We estimate system (2) using a 2SLS procedure; standard errors are double clustered by stock and day. ${ }^{17}$ As market quality measures we consider the logarithm of time-weighted bid depth and both the time-weighted relative spread and the time-weighted cent quoted spread. ${ }^{18}$

Tables $5-8$ report the results from the simultaneous equation model. We have four different specifications of system (2), which arise from the combination of the two different types of SPT (QJ and MID) and the two market quality measures (bid depth and relative spread). We are primarily interested in the $a_{1}$ and $b_{1}$ coefficients: $a_{1}$ measures the effect of SPT on market quality and $b_{1}$ measures the effect of market quality on SPT. The coefficients on our instruments, $a_{2}$ and $b_{2}$, are positive and highly significant. In other words, they appear to be good instruments. We present the results

\footnotetext{
${ }^{17}$ We repeat the estimation using a GMM approach allowing for heteroskedasticity of unknown form, still double clustering standard errors. The results are nearly identical.

${ }^{18}$ To economize space results for time-weighted cent quoted spread are presented in the Online Appendix.
} 
for the whole sample and for small and large capitalization stocks.

Results for the factors driving QJ $\left(b_{1}\right)$ are now consistent with Prediction 1 as QJ is positively related with the liquidity of the stock not only for depth, as in the Fama-MacBeth regressions, but also for the spread, measured both in cents and in basis points. Table 5 (Panel A) shows that $b_{1}$ is positive and statistically significant for depth, while Table 7 (Panel A) shows that it is negative and statistically significant for spread. For MID, we obtain similar results for depth (Table 6, Panel A) and spread (Table 8, Panel A).

Results for the effects of QJ on market quality show a positive effect on both depth and spread. The coefficient $a_{1}$ is positive and statistically significant in the depth system (Table 5, Panel A) and negative and statistically significant in the spread system (Table 7, Panel A). Interpreting market capitalization as a rough proxy of liquidity, we can see that consistently with Prediction 4 for liquid stocks (large cap) an increase in QJ determines an improvement of market quality measured by depth (Table 5, Panel A) and spread (Table 7, Panel A). By contrast, we cannot confirm this prediction for illiquid stocks as the results are not significant. Moreover, we do not observe any significant effect of MID on market quality (Table 6, Panel A, and Table 8, Panel A).

A word of caution is due, however, as we do not control for make-take fees. In practice, traders use dark markets to trade at sub-penny not only to step ahead of exiting limit orders at the top of regular exchanges but sometimes also to save on take fees. By internalizing orders a broker-dealer avoids paying the fees imposed by lit venues. Therefore, one should take the effects of different possible make-take fee structures into account both to correctly interpret our results, and to appropriately discuss our policy 
implications. $^{19}$

[Insert Tables 5-8 here]

\subsection{Robustness Check}

We rerun our simultaneous equation models including additional controls which are exogenous and can affect both market quality and SPT. In particular, following again Hasbrouck and Saar (2013), we include the daily return on SP500 and its volatility (proxied by VIX) to take into account market-wide activity. The same concern has been addressed by Brogaard, Hendershott and Riordan (2013) when studying the relation between HFT and volatility. Specifically, we estimate the following system of equations, which is a modification of system (2):

$$
\begin{aligned}
M Q M_{i, t} & =a_{1} S P_{i, t}+a_{2} M Q M_{N O T i, t}+a_{3} S P 500_{t}+a_{4} V I X_{t}+\varepsilon_{1, t} \\
S P_{i, t} & =b_{1} M Q M_{i, t}+b_{2} S P_{N O T i, t}+b_{3} S P 500_{t}+b_{4} V I X_{t}+\varepsilon_{2, t}
\end{aligned}
$$

We present the results in Tables $5-8$, Panel B. The results are robust to the inclusion of the additional controls.

\section{$7 \quad$ Policy Instruments and Regression Discontinuity Design}

In this Section we discuss our last empirical implication which aims at investigating which policy instrument regulators should use to influence QJ.

Our results from the Fama-MacBeth regressions show that when the stock price

\footnotetext{
${ }^{19}$ We cannot take into account make-take fees as for trades marked with the code "D" we cannot distinguish among different trading venues.
} 
increases the tick-to-price ratio decreases, and so does QJ. However, for the stocks included in the random sample representative of all listed stocks used for the FamaMacBeth regressions, SPT can take place only in ATSs. The objective of this Section is to verify the counterfactual. We investigate whether a reduction in the tick size would decrease QJ on ATSs in a framework in which QJ can also take place in regular exchanges. This allows us to verify whether traders undertake SPT in dark venues just because in lit markets this practice is banned or whether there are other reasons why traders choose to trade in the dark. We also investigate whether in this framework a reduction in the tick size is the adequate policy instrument to influence QJ.

To this end we exploit an additional feature of Rule 612 of Reg NMS which states that the minimum price improvement (tick size) changes from $\$ 0.01$ to $\$ 0.0001$ for stocks priced less than $\$ 1$. Given this cutoff, we employ a regression discontinuity design (RD). According to Cameron and Trivedi (2005), a RD is a quasi-experimental design in which the probability of receiving a treatment is a discontinuous function of one or more underlying variables. Such a design can arise in circumstances where a treatment is triggered by an administrative or organizational rule. This is exactly our case: since regulation imposes a cutoff at $\$ 1$, the treatment is having a price greater or equal to $\$ 1$ and so we can evaluate the effect of the treatment in the neighborhood of $\$ 1 .^{20}$ To sum up, the intuition behind a RD is that observations below and above the cutoff can be compared directly to draw inference on the effect of the treatment.

To run the RD we select a different sample than the one previously used. Our prior objective was to have a random sample representative of all NASDAQ and NYSE stocks. Now, we need stocks around $\$ 1$. So we take all NASDAQ listed stocks whose closing price at September 30, 2010 was between $\$ 0.8$ and $\$ 1.2$. Then, we restrict further our sample by retaining only the stocks whose price crosses the $\$ 1$ threshold at least once

\footnotetext{
${ }^{20}$ We will specify later what we are referring to with price.
} 
during the sample period (Table 1). The reason for applying this restriction is that identification of the treatment effect relies on stocks which cross the threshold (Lee and Lemieux, 2010).

The design is valid if the stock's price can be considered as good as randomly assigned below or above the threshold. We are dealing with the case of treatment assignment which is public knowledge (i.e, Rule 612 of Reg NMS). So, in principle, the limit orders (or the trades, depending on the assignment variable we are using) can crowd on one side of the cutoff. If it is the case that the assignment variable has been manipulated, the RD is not valid. However, in our case, the assumption of no manipulation seems to be legitimate since traders should not be able to move a stock price across the cutoff, so the assignment can be considered as good as randomly assigned. Nevertheless, as an additional check we run the density discontinuity test presented by McCrary (2008). The assignment variables are the bid price, the ask price and the execution price, depending on the specification we consider. We make this classification since also the Rule 612 of Reg NMS does. ${ }^{21}$ We run the test for the continuity of the assignment variables and we do not find any evidence of manipulation. We report the graphical representation of the test for the bid price in Figure 6. Results are analogous for ask price and execution price.

\section{[Insert Figure 6 here]}

We first study the effect of regulation on market quality (i.e., log of bid depth and relative spread). To build our dataset, for each stock and day we take a snapshot of the NBBO every hour starting from 9:30:00 AM. We estimate the following pooled OLS

\footnotetext{
${ }^{21}$ To be precise, Rule 612 of Reg NMS refers only to quotes. We extend the analysis to the price at which the trade has been executed.
} 
regression:

$$
y_{i t}=b_{0}+b_{1} D_{i, t}+b_{2}\left(\text { Price }_{i, t}-1\right)+b_{3}\left(\text { Price }_{i, t}-1\right) D_{i, t}+\varepsilon_{i, t}
$$

where Price $_{i, t}$ is the price rounded down to the closest cent. Price ${ }_{i, t}$ is reduced by one to locate the threshold at zero. $D_{i, t}$ is an indicator that takes the value of 1 if Price $_{i, t}$ is equal to or greater than $\$ 1$ and a value of 0 if Price $_{i, t}$ is less than $\$ 1$. $D_{i t}$ generates a discontinuity in the treatment around the threshold which allows for the estimation of the effect of the treatment. Index $i$ is for stock and index $t$ is for time. We include all the observations which satisfy the following condition: $-h \leq$ Price $_{i, t}-1 \leq h$, where $h$ is the bandwidth. We select a bandwidth equal to $\$ 0.10 .^{22}$ We estimate a pooled OLS regression even though we are dealing with a panel dataset. Indeed, in RD fixed effects are unnecessary for identification; it is sufficient to take into account the within-stock correlation of the errors over time using clustered standard errors (Lee and Lemieux, 2010). We present the results for the logarithm of bid depth and quoted spread percent as outcome variable $(y)$ and the bid price as assignment variable. The results of the estimation are in Tables 9 - 10 .

[Insert Tables 9 - 10 here]

The coefficients on the dummy variable $D_{i, t}$ show that for both ask depth and relative spread the discontinuity is statistically significant. Bid depth and relative spread both increase moving from below to above the $\$ 1$ cutoff. Therefore the implications for market quality are mixed: the inside spread improves but bid depth deteriorates. Interestingly, these conclusions are in line with the predictions of BRWW (2013) on the effects of a reduction of the tick size. A reduction in the tick size decreases traders' incentive to post

\footnotetext{
${ }^{22}$ We replicate the analysis also using the optimal bandwidth according to Imbens and Kalyanaraman (2012): the results still hold.
} 
limit orders and consequently it reduces market depth. It also reduces the inside spread as in today's fast markets traders active at the top of the LOB follow the downwards movement of the tick size and move towards the new narrower inside spread. Our results are robust when using 1.5 and 0.75 times the initial bandwidth $h$ (Tables 9 - 10) and when using the ask price as assignment variable. ${ }^{23}$ Note that the conclusion of the RD is valid only in the neighborhood of the cutoff and cannot be generalized to all stocks (this is why we have already studied the relation between SPT and market quality with other methods).

Besides the market quality measure, in this framework we also study the percentage of QJ as the outcome variable. ${ }^{24}$ In particular, we compute the percentage of QJ executed for each price bin of size one cent for each stock for the whole sample period with respect to the consolidated volume. To construct the price bin we round down all prices to the closest cent and we identify each bin with the lower bound. Then we estimate the following pooled OLS regression:

$$
Q J_{i, j}=b_{0}+b_{1} D_{i, j}+b_{2}\left(\text { PriceBin }_{i, j}-1\right)+b_{3}\left(\operatorname{PriceBin}_{i, j}-1\right) D_{i, j}+\varepsilon_{i, j}
$$

where PriceBin $_{i, j}$ is the lower bound of the penny bin. PriceBin ${ }_{i, j}$ is reduced by one to have the threshold at zero. $D_{i, j}$ is an indicator that takes the value of 1 if PriceBin $_{i, j}$ is equal to or greater than $\$ 1$ and a value of 0 if $\operatorname{PriceBin}_{i, j}$ is less than $\$ 1 . D_{i, j}$ generates a discontinuity in the treatment around the threshold which allows for the estimation of the effect of the treatment. Index $i$ is for stock and index $j$ is for price bin. We include all the observations which satisfy the following condition: $-h \leq \operatorname{Price}_{B_{i n}}-1 \leq h$, where $h$ is the bandwidth. We select a bandwidth equal to $\$ 0.10$. The results of the

\footnotetext{
${ }^{23}$ In this case the bid depth is substituted by the ask depth for consistency.

${ }^{24}$ As before, when talking about QJ we refer only to QJ executed on exchange "D".
} 
estimation are in Table 11.

[Insert Table 11 here]

Notwithstanding the fact that below the $\$ 1$ threshold QJ can be done also on lit markets, we observe a significant reduction when moving above the cutoff (i.e., coefficient of the dummy variable $D_{i, j}$ in Table 11). We can therefore conclude that a reduction in the tick size would not reduce QJ, but rather increase it. This result does not confirm our Prediction 5 as it is probably drawn by the advantage in terms of fees that dark markets may offer.

\section{Conclusions and Policy Implications}

During the last decade financial markets have been characterized by the growth of dark markets and fast trading. These two elements have fostered the development of sub-penny trading (SPT) which is a particular form of dark trading. SPT takes place when traders take advantage of the Sub-Penny Rule (Rule 612) and its exceptions by posting orders in the dark market or internalizing customers' orders at fractions of the tick size. In this way they gain price priority over the orders sitting at the inside quotes of regulated markets. Sub-penny orders are then executed against traders who demand liquidity by using smart order routing programs that allow them to hit the best quotes posted both in lit and in dark markets.

In this paper we show that in the U.S. approximately $10 \%$ of share volume executes at sub-penny increments due to queue-jumping (QJ). Approximately $3 \%$ executes exactly at the mid-quote, and could be the result either of QJ or of a midpoint cross in an opaque venue. These volumes are rapidly increasing and SPT is a concern to regulators as it can reduce the incentive for liquidity providers to post limit orders on regular 
exchanges and hence worsen market depth. For this reason in 2010 the SEC proposed the Trade-At rule to curtail dark trading, and more recently such rule has been introduced in Canada (October 2012) and in Australia (May 2013). In the 2010 concept release on market microstructure the SEC also opened the debate on SPT by asking questions on the factors that drive SPT, the effects of SPT on the quality of lit markets and more precisely on the adequate policy instrument to use to influence SPT. In this paper we answer most of these questions.

We find that SPT varies significantly by listing exchange, as QJ is significantly higher for NASDAQ than for NYSE listed stocks. Consistently with a recent theoretical model by Buti, Rindi, Wen and Werner (BRWW, 2013), we show that QJ is positively related to depth and negatively related to stock price. This means that broker-dealers use dark markets for QJ especially for stocks where competition for the provision of liquidity is high and hence it is difficult to gain price priority on lit platforms. The use of QJ is also intense in low priced stocks for which the profit from price improvement is higher relative to the asset value.

We find that SPT improves both depth and spread, especially for large capitalization stocks. This is also consistent with BRWW (2013) predictions. We do not find that SPT harms liquidity even though we do not investigate whether market participants benefit or are harmed by SPT. We leave this interesting topic for future research.

Our analysis also allows us to draw conclusions on the efficacy of the tick size as a policy instrument to influence SPT. In the U.S. Rule 612 sets the minimum price improvement of 1 penny only for stocks priced above $\$ 1$, while it permits executions at sub-penny increments for stocks priced below $\$ 1$. We exploit this feature of the Sub-penny Rule to conduct a regression discontinuity analysis and study what happens to QJ as the price of a stock moves across the trigger point $(\$ 1.00)$ below which the main market is also allowed to quote and trade in sub-penny increments. We obtain 
two results.

We first show that surprisingly QJ increases for stocks priced below $\$ 1$, which means that if regulators decreased the tick size to allow QJ for all stocks, traders would not move back to lit markets. We conjecture that the make-take pricing structure of lit vs dark markets could play a role in traders' preference for dark venues. There might be an interaction between the smaller tick size associated with stocks priced below $1 \$$, the activity of market making/HFT and the resulting QJ. The so called "Knight event" of August 1, 2012 is reminiscent of the interaction of these activities, and by looking at the percentage of QJ in the window July 26 - August 7 (symmetric around the day of the event) we observe a substantial drop in QJ (Figure 5). ${ }^{25}$

Second, we show that the inside spread improves and the depth deteriorates when a stock moves from above to below the $\$ 1$ threshold. This means that consistently with BRWW (2013) a reduction in the tick size would have a detrimental effect on the provision of liquidity: not only depth would decrease, but by reducing spreads a smaller tick size would further curtail broker-dealers' profits and hence potentially decrease rather than increase their incentive to supply liquidity. We conclude that the tick size does not appear to be the appropriate policy instrument to use if the objective is to reduce $\mathrm{SPT}$.

\footnotetext{
${ }^{25}$ On August 1, 2012 the Knight Capital Group is believed to have accidentally released their new market making software into the NYSE's system. This happened when the market opened and in 45 minutes Knight Capital unintentionally acquired a $\$ 3.5$ billion net long position in 80 stocks and $\$ 3.15$ billion net short position in 74 stocks. An algo, not able to 'read' the accumulated fills, kept sending orders to market (TABB, 15 November 2013). By 10 AM the test software was killed. In Figure 5 we insert the daily average of QJ for the group of our NYSE sample stocks and for the 13 stocks that achieved the minimum QJ on August 1.
} 


\section{References}

Brogaard, J., T. Hendershott, and R. Riordan, 2013. High Frequency Trading and Price Discovery. Review of Financial Studies, forthcoming.

Buti, S. and B. Rindi, 2013. Undisclosed Orders and Optimal Submission Strategies in a Limit Order Market. Journal of Financial Economics, 109, 3, 797-812.

Buti, S., B. Rindi, and I. M. Werner, 2011. Diving into Dark Pools. Working paper. Fisher College of Business.

Buti, S., B. Rindi, Y. Wen and I. M. Werner, 2013. Tick Size Regulation and Sub-Penny Trading, Working paper. Fisher College of Business.

Cameron, A. C., and P. K. Trivedi, 2005. Microeconometrics: Methods and Applications, Cambridge University Press.

Comerton-Forde, C. and T. J. Putnins, 2013. Dark Trading and Price Discovery. Woking paper.

Degryse, H., F. de Jong and V. van Kervel, 2011. The impact of dark and visible fragmentation on market quality. TILEC Discussion Paper.

Foucault, T., 1999. Order flow composition and trading costs in a dynamic limit order market. Journal of Financial Markets 2, 99-134.

Foucault, T., O. Kadan, and E. Kandel. 2005. Limit Order Book as A Market for Liquidity. Review of Financial Studies 18:1171-1217.

Goettler, R. L., C.A. Parlour and U. Rajan, 2005. Equilibrium in a dynamic limit order market. Journal of Finance 60, 2149-2192.

Hasbrouck, J., and G. Saar, 2013. Low Latency Trading. Journal of Financial Markets, forthcoming.

Hatheway, F., A. Kwan, and H. Zheng, 2013. An Empirical Analysis of Market Segmentation on U.S. Equities Markets. Working Paper, SSRN FEN Library. 
Imbens, G., and K. Kalyanaraman, 2012. Optimal Bandwidth Choice for the Regression Discontinuity Estimator. The Review of Economic Studies 79: 933-959.

Kwan, A., R. Masulis, and T. McInish, 2013. Trading rules, competition for order flows and market fragmentation. Working Paper, SSRN FEN Library.

Lee, C., and M. Ready, 1991. Inferring Trade Direction from Intraday Data. Journal of Finance 46: 733-746.

Lee, D. S., and T. Lemieux, 2010. Regression Discontinuity Designs in Economics. Journal of Economic Literature 48(2): 281-355.

McCrary, J., 2008. Manipulation of the Running Variable in the Regression Discontinuity Design: A Density Test. Journal of Econometrics 142: 698-714.

Nimalendran, M. and S. Ray, 2012. Informational Linkages Between Dark and Lit Trading Venues. Working paper, University of Florida.

Parlour, C. A., 1998. Price Dynamics in Limit Order Markets. Review of Financial Studies 11, 789-816.

Ready, M., 2013. Determinants of Volume in Dark Pools.Working paper. University of Wisconsin.

Rosenblatt Securities Inc., 2013. Trading Talk, Let there be light, February 26.

Rosu, I., 2009. A dynamic model of the limit order book. Review of Financial Studies $22,4601-4641$.

U.S. Securities and Exchange Commission (SEC), 2010. Concept Release on Equity Market Structure No. 34-61358.

U.S. Securities and Exchange Commission (SEC), 2012. Report to Congress on Decimalization. 
Figure 1: NASDAQ queue-jumping and mid-crossing versus stock price

This figure reports the queue-jumping and mid-crossing percentage over the sample period for each NASDAQ stock against the price of the stock.

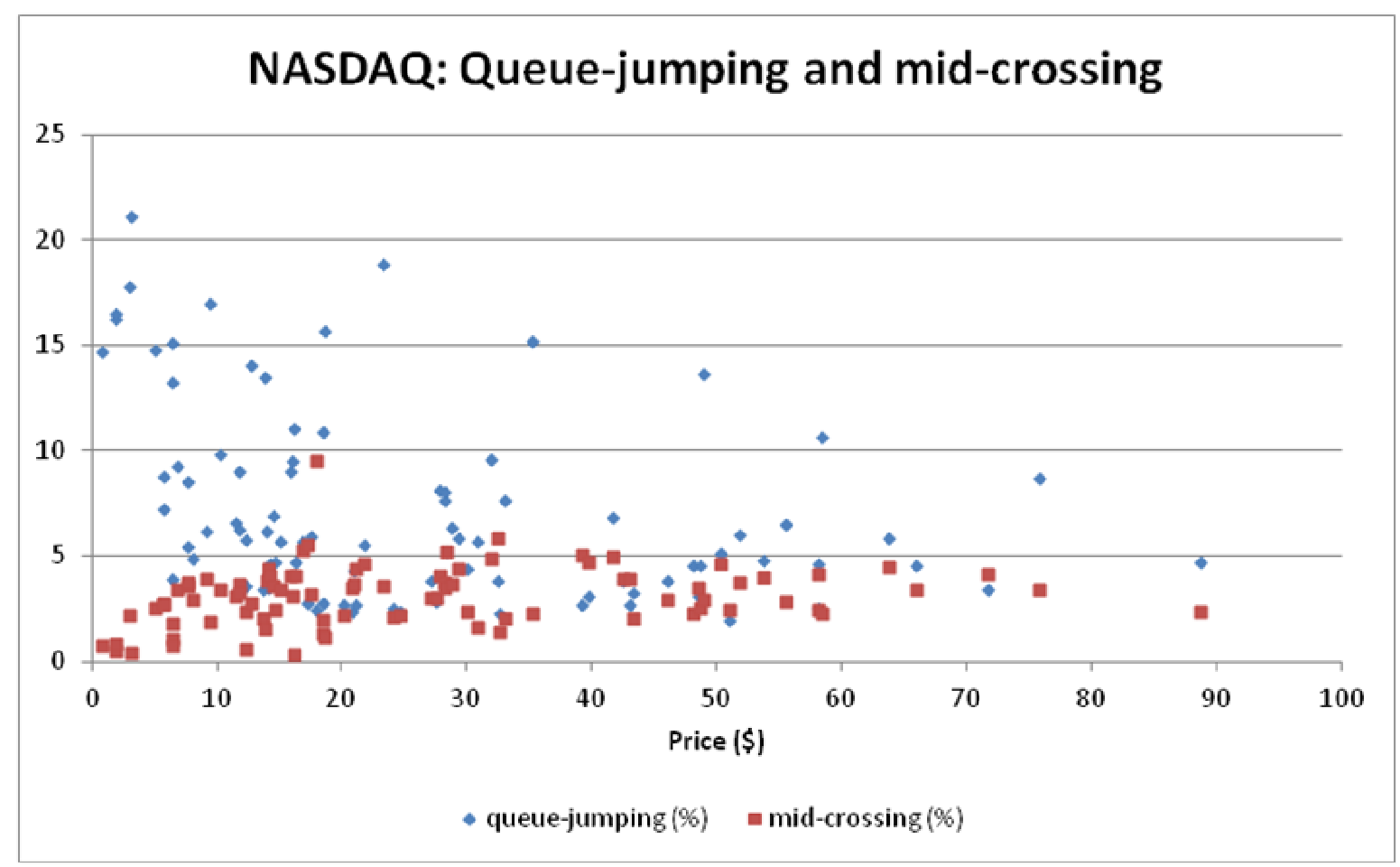


Figure 2: NYSE queue-jumping and mid-crossing versus stock price

This figure reports the queue-jumping and mid-crossing percentage over the sample period for each NYSE stock against the price of the stock.

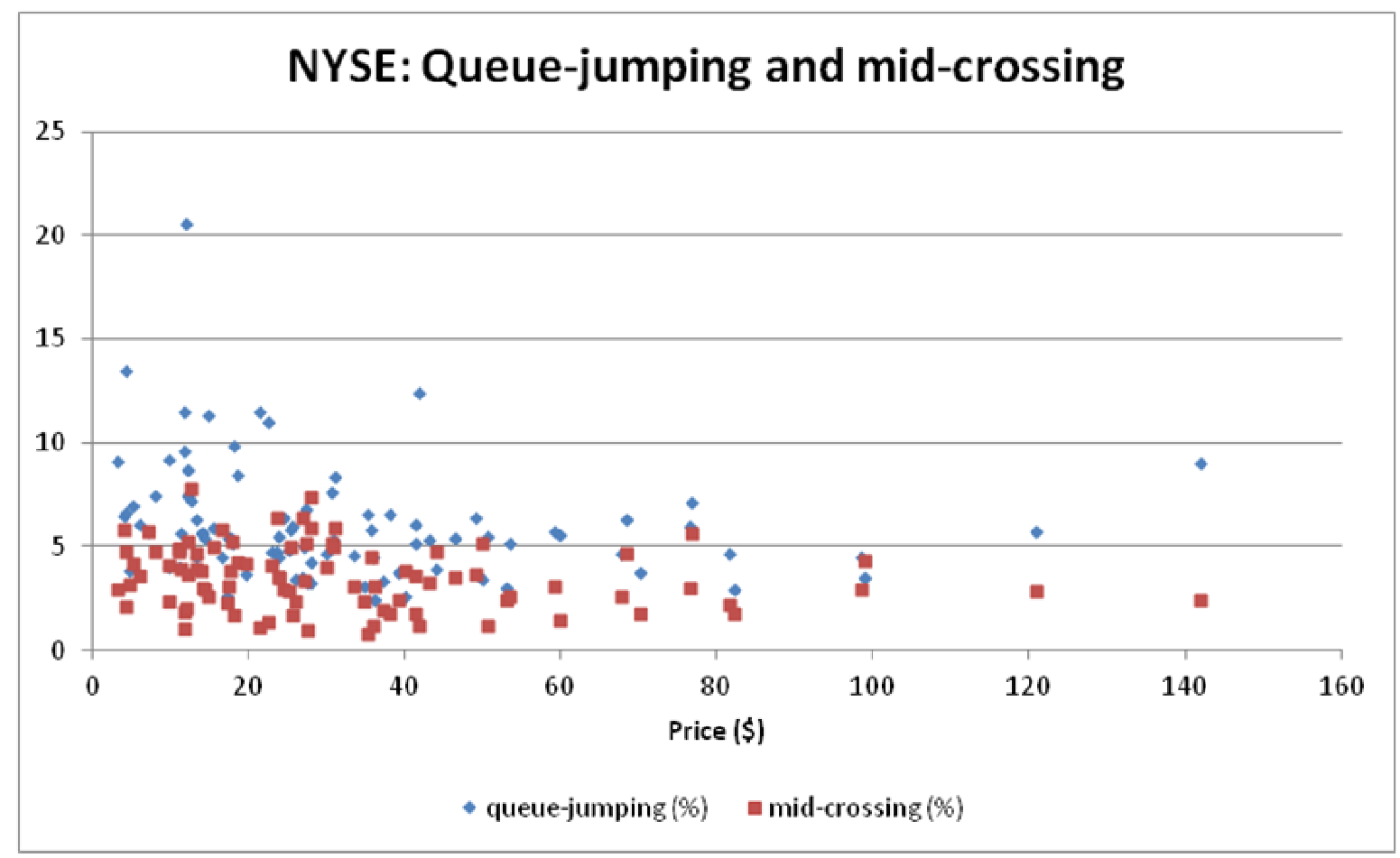


Figure 3: NASDAQ distribution of sub-penny (including mid-crossing)

This figure reports the sub-penny in percentage of consolidated volume for each bin over the sample period and for the group of 30 high-priced NASDAQ stocks.

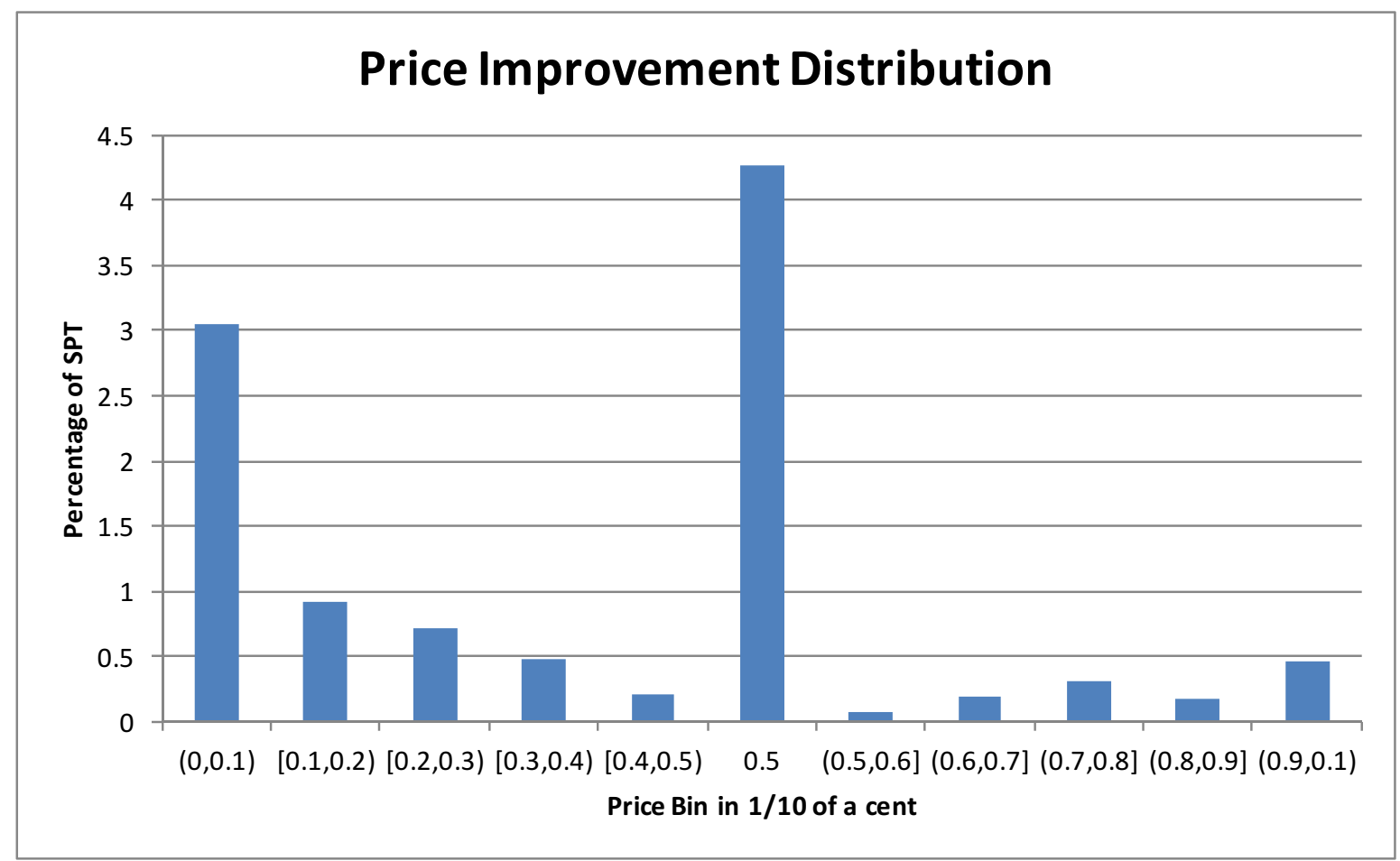


Figure 4: NYSE distribution of sub-penny (including mid-crossing)

This figure reports the sub-penny in percentage of consolidated volume for each bin over the sample period and for the group of 30 high-priced NYSE stocks.

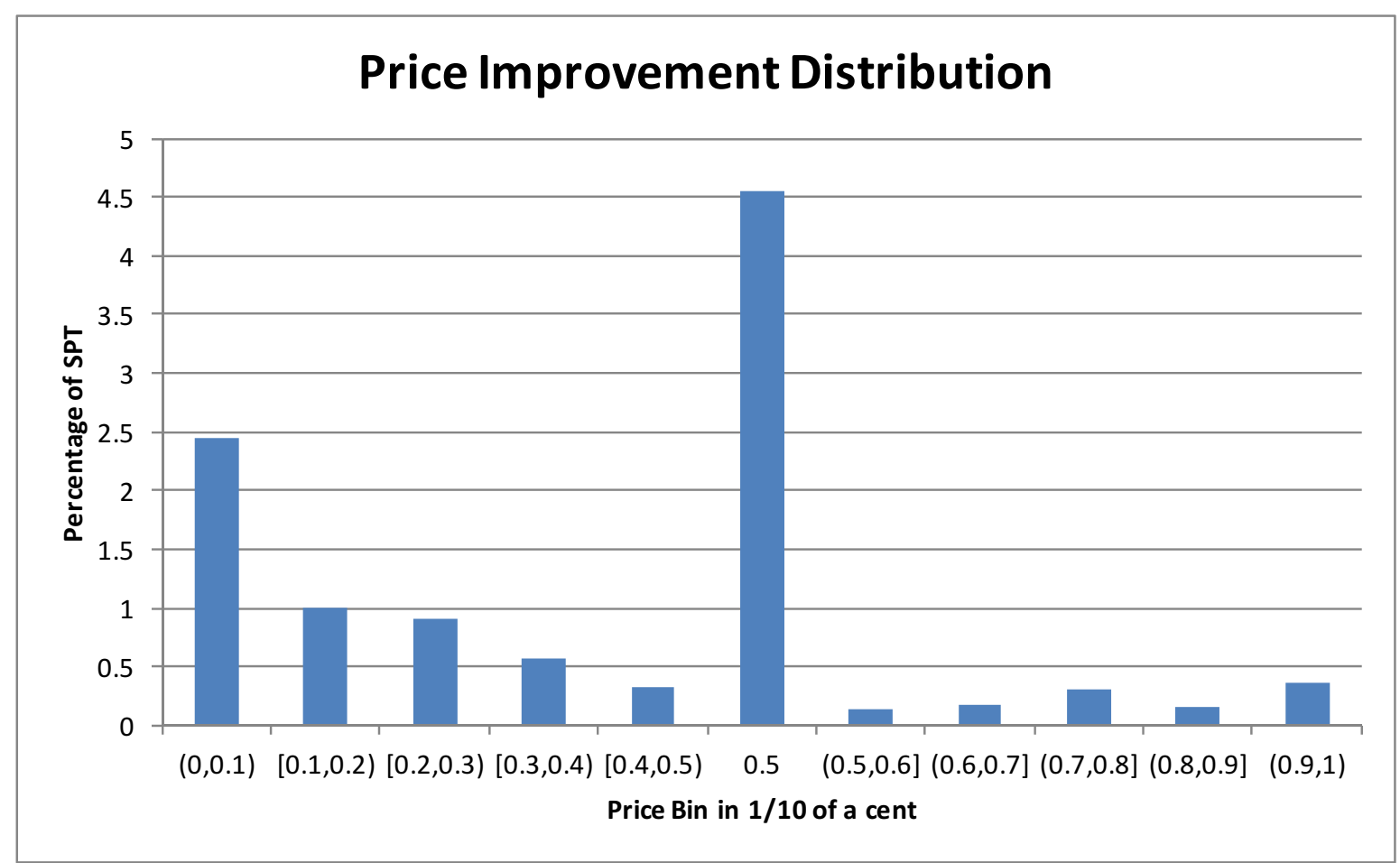


Figure 5: Time series of QJ around the Knight event (August 1, 2012)

This figure reports the time series of QJ for a sample of 83 NYSE listed stocks (the survivors from the original sample of 90). We have 9 daily observations of cross-sectional averages of QJ. The midpoint of the series corresponds to the Knight event. We take a window of 4 days before and after the event. The dashed line is the time-series of the whole sample, while the solid line is the time-series of stocks which achieve the minimum QJ in correspondence of August 1, 2012.

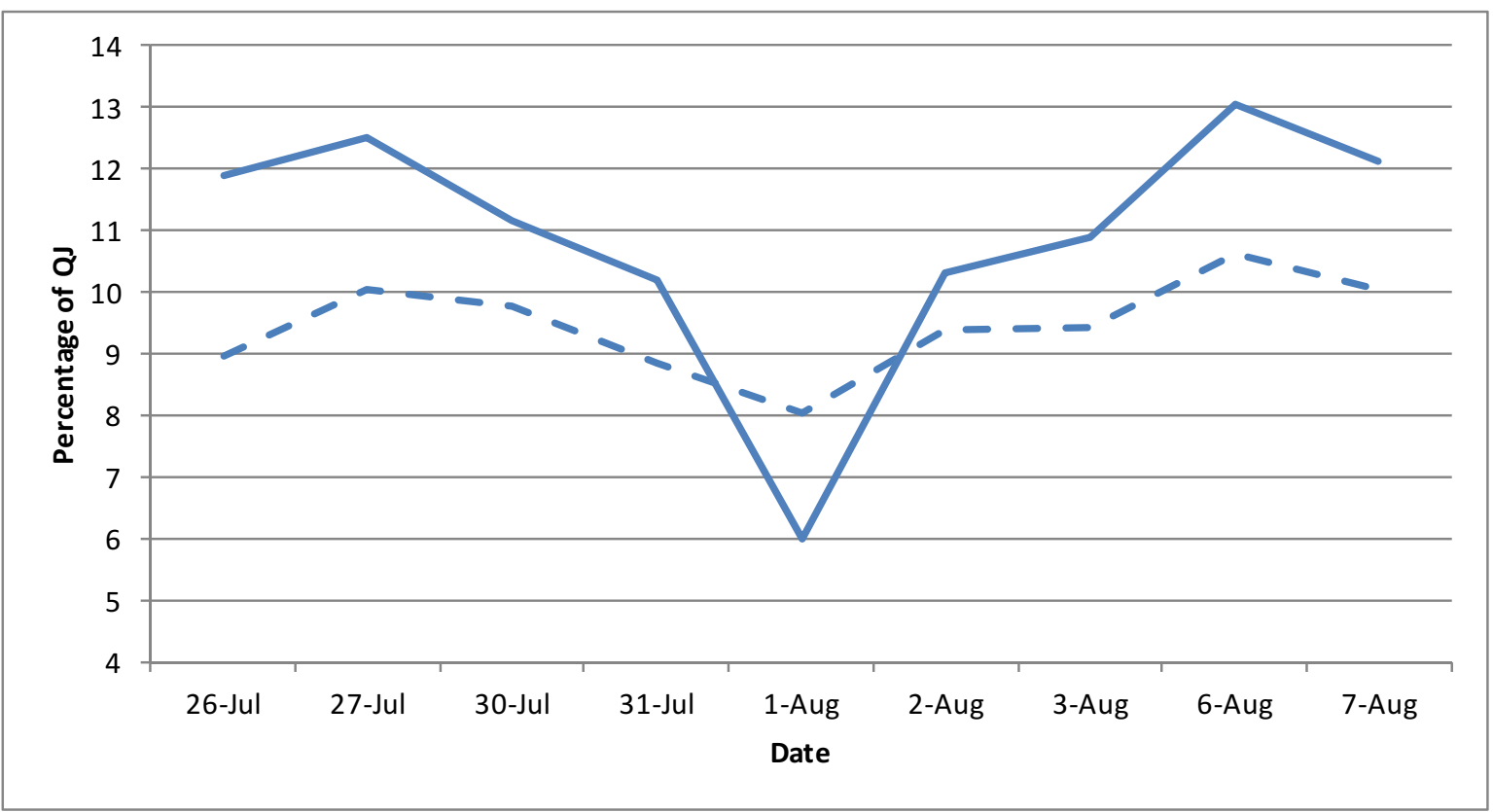


Figure 6: Density Representation for bid price

This figure reports the density of the assignment variable (i.e., bid price) to assess the continuity of the variable itself across the cutoff. The points represents the smoothed histogram while the solid black line represents the smoothed density with confidence bands (solid grey lines). Both histogram and density are smoothed using a triangle kernel.

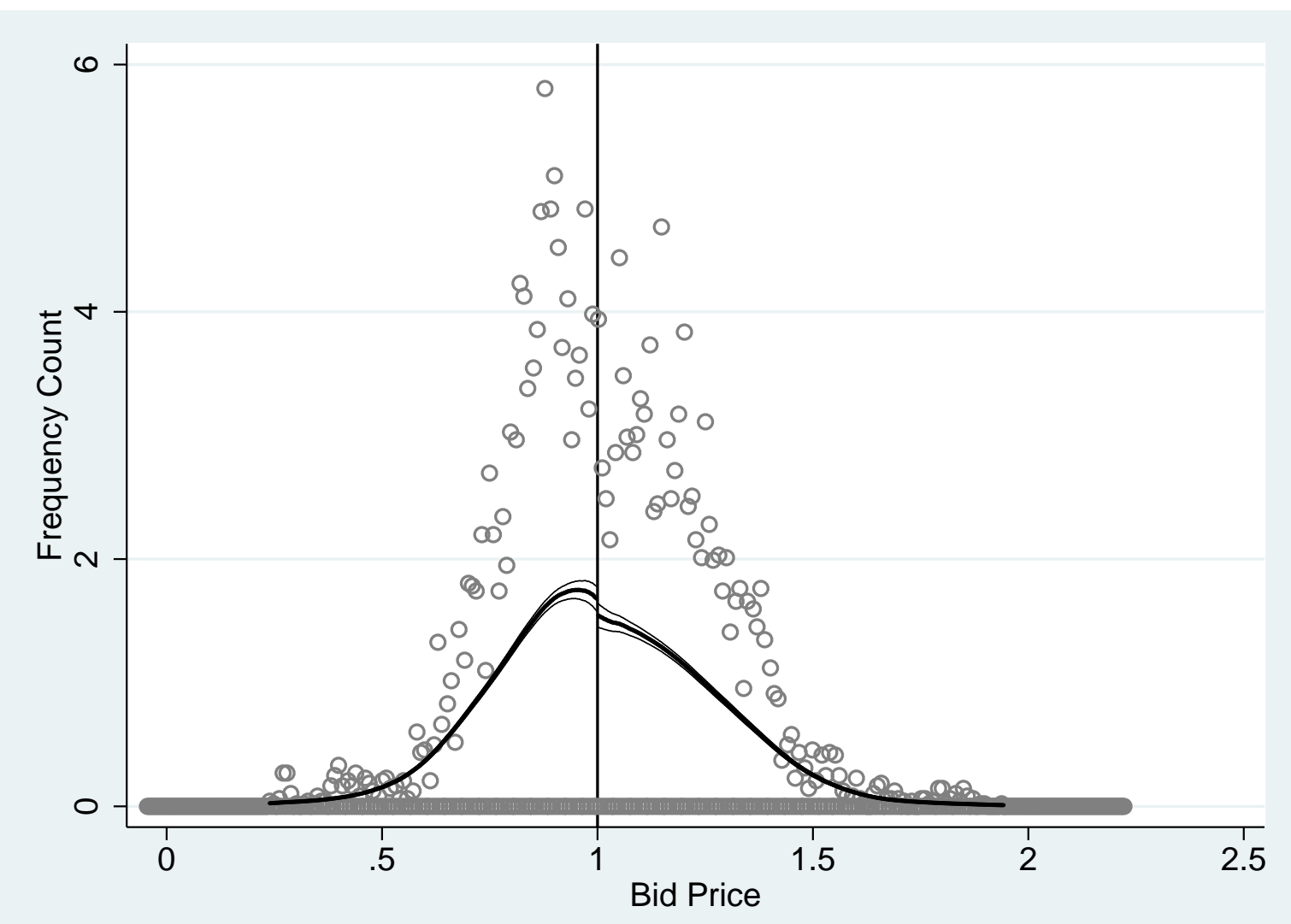


Table 1: Stocks descriptive statistics

The sample period is October 1, 2010 - November 30, 2010. All variables are daily (timeweighted) averages. Market cap is the stock's market capitalization in millions from CRSP. Closing price is in dollar from CRSP. Relative spread is the difference between the bid and ask price over the midquote. Bid depth is the bid depth at NBBO in unit of trade (100 shares). Price range is the difference between the maximum and the minimum price over the maximum price. Queue-jumping is the QJ on D computed as the ratio of volume executed in QJ over the total consolidated volume. Mid-crossing is the MID on D computed as the ratio of volume executed in MID over the total consolidated volume. Sub-penny is the SPT on D computed as the ratio of volume executed in SPT over the total consolidated volume. Statistics are reported for the full sample (NASDAQ and NYSE) and for the NASDAQ and the NYSE separately. Furthermore, for each panel, we report the summary statistics for the three market capitalization groups (SMALL, MEDIUM and LARGE). Finally, we report statistics for the sample of NASDAQ stocks around $\$ 1$, splitted between above and below $\$ 1$, except for statistics which are computed daily (i.e., market capitalization, closing price and price range).

\begin{tabular}{|c|c|c|c|c|c|c|c|c|}
\hline & $\begin{array}{l}\text { Market Cap } \\
(\text { Million \$) }\end{array}$ & $\begin{array}{c}\text { Closing Price } \\
(\$)\end{array}$ & $\begin{array}{c}\text { Time-weighted } \\
\text { relative spread }(\% / 000)\end{array}$ & $\begin{array}{l}\text { Time-weighted } \\
\text { bid depth (UoT) }\end{array}$ & $\begin{array}{c}\text { Price range } \\
(\%)\end{array}$ & $\begin{array}{c}\text { Queue-jumping } \\
\text { on D (\%) }\end{array}$ & $\begin{array}{c}\text { Mid-crossing } \\
\text { on D }(\%)\end{array}$ & $\begin{array}{l}\text { Sub-penny } \\
\text { on D (\%) }\end{array}$ \\
\hline NASDAQ+NYSE & 7324.30 & 29.70 & 0.149 & 14398.67 & 0.04 & 6.59 & 3.34 & 9.93 \\
\hline Small & 534.48 & 26.78 & 0.272 & 957.70 & 0.05 & 7.68 & 2.51 & 10.19 \\
\hline Medium & 1740.48 & 29.10 & 0.112 & 2321.72 & 0.03 & 5.49 & 3.40 & 8.89 \\
\hline Large & 19697.94 & 33.22 & 0.062 & 39916.59 & 0.02 & 6.61 & 4.10 & 10.71 \\
\hline NASDAQ & 5807.27 & 26.58 & 0.153 & 3766.59 & 0.03 & 7.16 & 3.16 & 10.32 \\
\hline Small & 462.41 & 22.75 & 0.287 & 1439.62 & 0.04 & 9.11 & 2.11 & 11.23 \\
\hline Medium & 1648.04 & 26.92 & 0.111 & 2700.43 & 0.03 & 5.79 & 3.51 & 9.30 \\
\hline Large & 15311.36 & 30.05 & 0.061 & 7159.73 & 0.02 & 6.57 & 3.86 & 10.43 \\
\hline NYSE & 8841.33 & 32.83 & 0.145 & 25030.75 & 0.04 & 6.03 & 3.51 & 9.54 \\
\hline Small & 606.55 & 30.81 & 0.257 & 475.77 & 0.05 & 6.25 & 2.90 & 9.15 \\
\hline Medium & 1832.93 & 31.28 & 0.114 & 1943.02 & 0.03 & 5.18 & 3.29 & 8.48 \\
\hline Large & 24084.53 & 36.40 & 0.064 & 72673.45 & 0.02 & 6.65 & 4.35 & 11.00 \\
\hline NASDAQ around $\$ 1$ & 34.28 & 0.90 & & & 0.10 & & & \\
\hline Below & & & 0.011 & 718.18 & & 2.13 & 30.04 & 32.17 \\
\hline Above & & & 0.013 & 6948.27 & & 0.53 & 13.64 & 14.17 \\
\hline
\end{tabular}

Table 2: Price improvement calculation

Hereafter an example of price improvement calculation.

\begin{tabular}{|c|c|c|c|c|c|c|}
\hline SYMBOL & DATE & TIME & PRICE & TRADE SIGN & PRICE ROUNDED & PRICE IMPROVEMENT \\
\hline ASEI & 20101018 & $10: 13: 12$ & 78.7501 & BUY & 78.75 & 0.0001 \\
\hline ASEI & 20101018 & $10: 25: 46$ & 78.7975 & SELL & 78.8 & 0.0025 \\
\hline
\end{tabular}




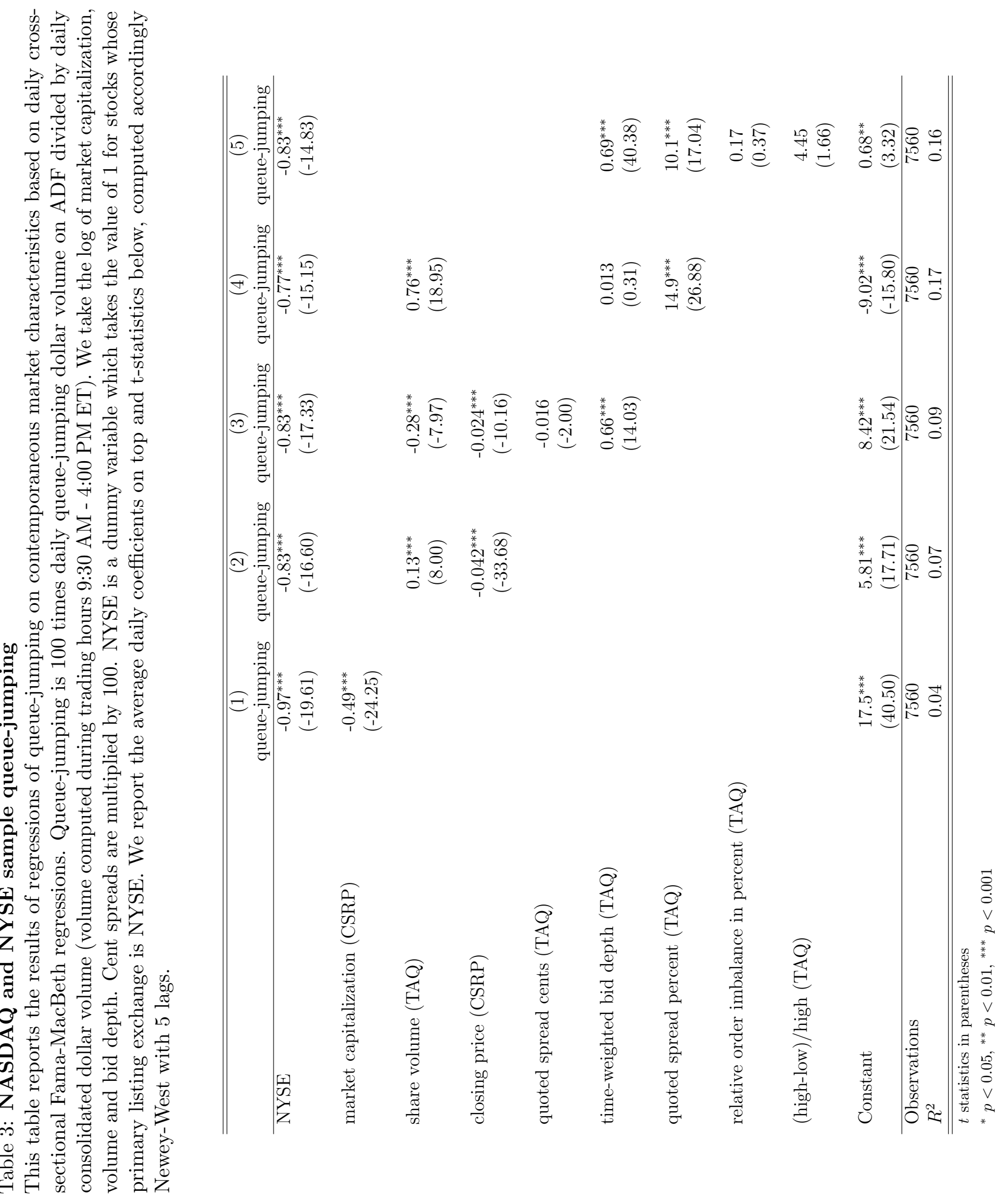




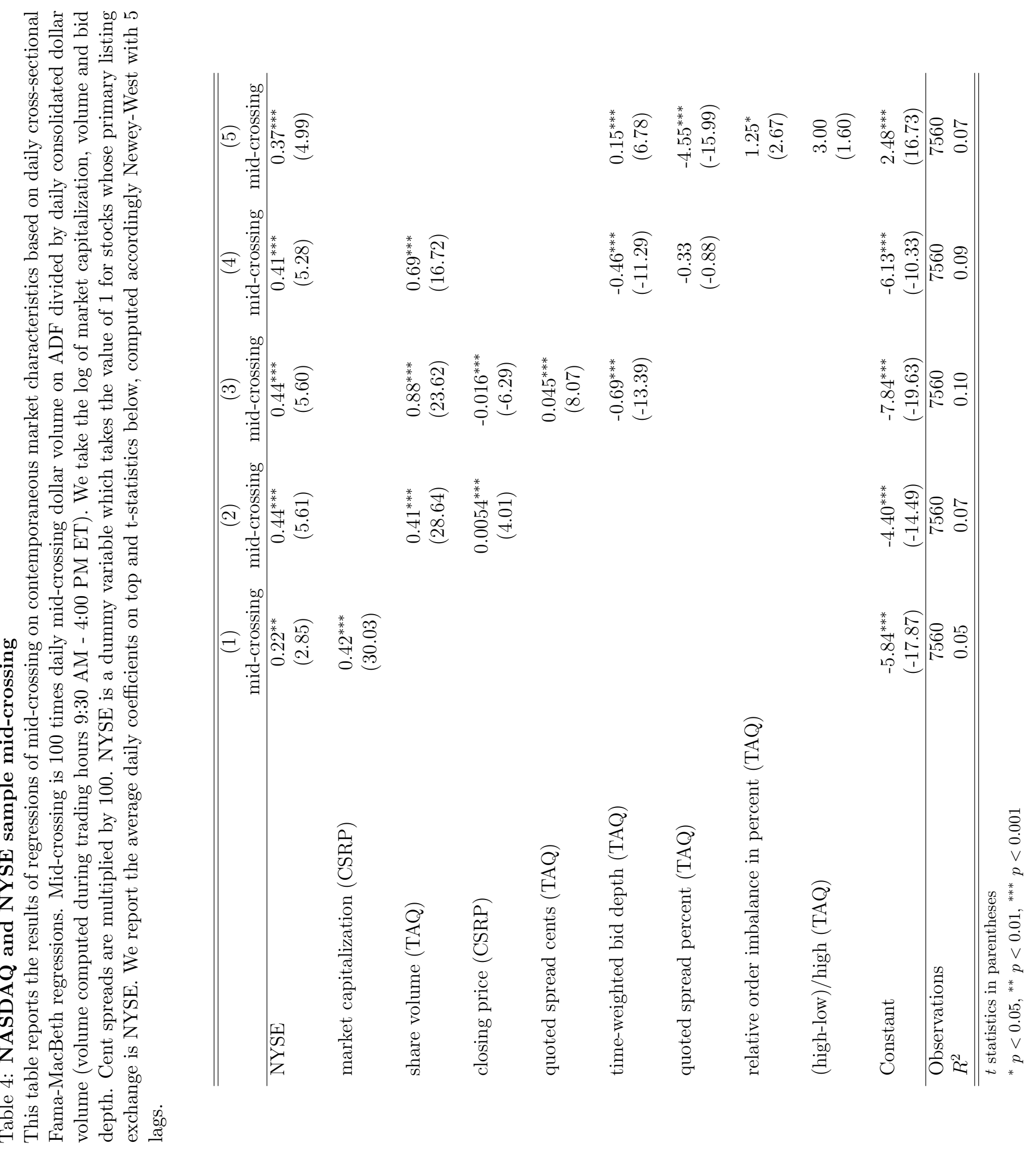


Table 5: NASDAQ and NYSE queue-jumping and time-weighted bid depth

This table reports the results from the analysis of the relationship between queue-jumping activity and time-weighted bid depth. We measure queue-jumping activity as QJ, which is defined as 100 times daily queue-jumping dollar volume on ADF divided by daily consolidated dollar volume (volume computed during trading hours 9:30 AM - 4:00 PM ET). Due to the potential simultaneity between time-weighted bid depth (log of) and queue-jumping activity, in Panel A we estimate the following two-equation simultaneous model for QJ and the log of time-weighted bid depth (MQM):

$M Q M_{i, t}=a_{1} Q J_{i, t}+a_{2} M Q M_{N O T i, t}+\varepsilon_{1, t}$

$Q J_{i, t}=b_{1} M Q M_{i, t}+b_{2} Q J_{N O T i, t}+\varepsilon_{2, t}$

In Panel B, we estimate the following two-equation simultaneous model, including as control, the return on SP500 and the VIX:

$M Q M_{i, t}=a_{1} Q J_{i, t}+a_{2} M Q M_{N O T i, t}+a_{3} S P 500_{t}+a_{4} V I X_{t}+\varepsilon_{1, t}$

$Q J_{i, t}=b_{1} M Q M_{i, t}+b_{2} Q J_{N O T i, t}+b_{3} S P 500_{t}+b_{4} V I X_{t}+\varepsilon_{2, t}$

As an instrument for $Q J_{i, t}$ we use $Q J_{N O T i, t}$, which is the daily average QJ activity of other stocks listed on the same exchange, in the same market capitalization grouping (LARGE, MEDIUM, SMALL)(excluding stock i). Similarly, as an instrument for $M Q M_{i, t}$ we use $M Q M_{N O T i, t}$, which is the average time-weighted bid depth (log of) for other stocks listed on the same exchange, in the same market capitalization grouping (LARGE, MEDIUM, SMALL)(excluding stock i). We estimate the simultaneous equation model by pooling observations across all stocks and days in the sample. To make the pooling meaningful, we de-mean all variables by deducting the stock-specific average and scale all variables by dividing by the stock-specific standard deviation to control for stock fixed effects. We report the estimated coefficients on top and p-values below. Estimation is done with 2SLS with two-way clustered standard errors (i.e., stock and day).

\begin{tabular}{|c|c|c|c|c|c|c|}
\hline & \multicolumn{3}{|c|}{ Panel A } & \multicolumn{3}{|c|}{ Panel B } \\
\hline & (1) & (2) & (3) & (4) & (5) & (6) \\
\hline & Full sample & Small & Large & Full sample & Small & Large \\
\hline \multirow[t]{2}{*}{$a_{1}$} & $0.34^{* * *}$ & 0.044 & $0.28^{*}$ & $0.32^{* * *}$ & 0.055 & $0.25^{*}$ \\
\hline & $(0.000)$ & $(0.802)$ & $(0.022)$ & $(0.000)$ & $(0.738)$ & $(0.032)$ \\
\hline \multirow[t]{2}{*}{$a_{2}$} & $0.52^{* * *}$ & $0.37^{* * *}$ & $0.64^{* * *}$ & $0.52^{* * *}$ & $0.36^{* * *}$ & $0.62^{* * *}$ \\
\hline & $(0.000)$ & $(0.000)$ & $(0.000)$ & $(0.000)$ & $(0.000)$ & $(0.000)$ \\
\hline \multirow[t]{2}{*}{$a_{3}$} & & & & 0.015 & -0.017 & 0.035 \\
\hline & & & & $(0.295)$ & $(0.521)$ & $(0.193)$ \\
\hline$a_{4}$ & & & & $\begin{array}{c}0.000025 \\
(0.965)\end{array}$ & $\begin{array}{c}0.000090 \\
(0.928)\end{array}$ & $\begin{array}{c}-0.000042 \\
(0.968)\end{array}$ \\
\hline Observations & 7560 & 2520 & 2520 & 7560 & 2520 & 2520 \\
\hline \multirow[t]{2}{*}{$b_{1}$} & $0.28^{* *}$ & 0.058 & $0.25^{*}$ & $0.26^{* * *}$ & 0.071 & $0.23^{*}$ \\
\hline & $(0.001)$ & $(0.818)$ & $(0.035)$ & $(0.001)$ & $(0.776)$ & $(0.024)$ \\
\hline \multirow[t]{2}{*}{$b_{2}$} & $0.42^{* * *}$ & $0.42^{* * *}$ & $0.55^{* * *}$ & $0.41^{* * *}$ & $0.41^{* * *}$ & $0.54^{* * *}$ \\
\hline & $(0.000)$ & $(0.000)$ & $(0.000)$ & $(0.000)$ & $(0.000)$ & $(0.000)$ \\
\hline \multirow[t]{2}{*}{$b_{3}$} & & & & $0.026^{*}$ & 0.016 & 0.018 \\
\hline & & & & $(0.045)$ & $(0.505)$ & (0.499) \\
\hline \multirow[t]{2}{*}{$b_{4}$} & & & & -0.00024 & -0.00015 & -0.00023 \\
\hline & & & & $(0.628)$ & $(0.885)$ & $(0.812)$ \\
\hline Observations & 7560 & 2520 & 2520 & 7560 & 2520 & 2520 \\
\hline
\end{tabular}


Table 6: NASDAQ and NYSE mid-crossing and time-weighted bid depth

This table reports the results from the analysis of the relationship between mid-crossing activity and time-weighted bid depth. We measure mid-crossing activity as MID, which is defined as 100 times daily mid-crossing dollar volume on ADF divided by daily consolidated dollar volume (volume computed during trading hours 9:30 AM - 4:00 PM ET). Due to the potential simultaneity between time-weighted bid depth (log of) and mid-crossing activity, in Panel A we estimate the following two-equation simultaneous model for MID and the log of time-weighted bid depth (MQM):

$M Q M_{i, t}=a 1 * M I D_{i, t}+a 2 * M Q M_{N O T i, t}+\varepsilon_{1, t}$

$Q J_{i, t}=b 1 * M Q M_{i, t}+b 2 * M I D_{N O T i, t}+\varepsilon_{2, t}$

In Panel B, we estimate the following two-equation simultaneous model, including as control, the return on SP500 and the VIX:

$M Q M_{i, t}=a_{1} M I D_{i, t}+a_{2} M Q M_{N O T i, t}+a_{3} S P 500_{t}+a_{4} V I X_{t}+\varepsilon_{1, t}$

$M I D_{i, t}=b_{1} M Q M_{i, t}+b_{2} M I D_{N O T i, t}+b_{3} S P 500_{t}+b_{4} V I X_{t}+\varepsilon_{2, t}$

As an instrument for $M I D_{i, t}$ we use $M I D_{N O T i, t}$, which is the daily average MID activity of other stocks listed on the same exchange, in the same market capitalization grouping (LARGE, MEDIUM, SMALL)(excluding stock i). Similarly, as an instrument for $M Q M_{i, t}$ we use $M Q M_{N O T i, t}$, which is the average time-weighted bid depth (log of) for other stocks listed on the same exchange, in the same market capitalization grouping (LARGE, MEDIUM, SMALL)(excluding stock i). We estimate the simultaneous equation model by pooling observations across all stocks and days in the sample. To make the pooling meaningful, we de-mean all variables by deducting the stock-specific average and scale all variables by dividing by the stock-specific standard deviation to control for stock fixed effects. We report the estimated coefficients on top and p-values below. Estimation is done with 2SLS with two-way clustered standard errors (i.e., stock and day).

\begin{tabular}{|c|c|c|c|c|c|c|}
\hline & \multicolumn{3}{|c|}{ Panel A } & \multicolumn{3}{|c|}{ Panel B } \\
\hline & (1) & $(2)$ & (3) & (4) & (5) & (6) \\
\hline & Full sample & Small & Large & Full sample & Small & Large \\
\hline \multirow[t]{2}{*}{$a_{1}$} & 0.20 & -0.041 & 0.25 & 0.16 & -0.046 & 0.16 \\
\hline & $(0.376)$ & $(0.889)$ & (0.439) & (0.494) & $(0.872)$ & $(0.644)$ \\
\hline \multirow[t]{2}{*}{$a_{2}$} & $0.60^{* * *}$ & $0.37^{* * *}$ & $0.72^{* * *}$ & $0.59 * * *$ & $0.36^{* * *}$ & $0.69^{* * *}$ \\
\hline & $(0.000)$ & $(0.000)$ & $(0.000)$ & $(0.000)$ & $(0.000)$ & $(0.000)$ \\
\hline \multirow[t]{2}{*}{$a_{3}$} & & & & $0.030^{*}$ & -0.016 & 0.044 \\
\hline & & & & $(0.028)$ & $(0.521)$ & $(0.088)$ \\
\hline \multirow{2}{*}{$a_{4}$} & & & & -0.000094 & 0.000080 & -0.00014 \\
\hline & & & & $(0.870)$ & $(0.935)$ & $(0.889)$ \\
\hline Observations & 7560 & 2520 & 2520 & 7560 & 2520 & 2520 \\
\hline \multirow[t]{2}{*}{$b_{1}$} & $0.11^{*}$ & -0.055 & 0.15 & 0.096 & -0.060 & 0.12 \\
\hline & $(0.045)$ & $(0.804)$ & $(0.086)$ & (0.095) & $(0.797)$ & $(0.230)$ \\
\hline \multirow[t]{2}{*}{$b_{2}$} & $0.25^{* * *}$ & $0.28^{* * *}$ & $0.28^{* * *}$ & $0.24^{* * *}$ & $0.28^{* * *}$ & $0.26^{* * *}$ \\
\hline & $(0.000)$ & $(0.000)$ & $(0.000)$ & (0.000) & $(0.000)$ & (0.001) \\
\hline \multirow[t]{2}{*}{$b_{3}$} & & & & 0.016 & -0.0060 & 0.032 \\
\hline & & & & $(0.270)$ & (0.793) & $(0.249)$ \\
\hline \multirow[t]{2}{*}{$b_{4}$} & & & & -0.000081 & 0.00010 & -0.00020 \\
\hline & & & & $(0.886)$ & $(0.914)$ & $(0.810)$ \\
\hline Observations & 7560 & 2520 & 2520 & 7560 & 2520 & 2520 \\
\hline
\end{tabular}


Table 7: NASDAQ and NYSE queue-jumping and time-weighted relative spread This table reports the results from the analysis of the relationship between queue-jumping activity and time-weighted relative spread. We measure mid-crossing activity as QJ, which is defined as 100 times daily queue-jumping dollar volume on ADF divided by daily consolidated dollar volume (volume computed during trading hours 9:30 AM - 4:00 PM ET). Due to the potential simultaneity between time-weighted relative spread and queue-jumping activity, in panel A we estimate the following two-equation simultaneous model for QJ and the log of time-weighted relative spread (MQM):

$M Q M_{i, t}=a_{1} Q J_{i, t}+a_{2} M Q M_{N O T i, t}+\varepsilon_{1, t}$

$Q J_{i, t}=b_{1} M Q M_{i, t}+b_{2} Q J_{N O T i, t}+\varepsilon_{2, t}$

In Panel B, we estimate the following two-equation simultaneous model, including as control, the return on SP500 and the VIX:

$M Q M_{i, t}=a_{1} Q J_{i, t}+a_{2} M Q M_{N O T i, t}+a_{3} S P 500_{t}+a_{4} V I X_{t}+\varepsilon_{1, t}$

$Q J_{i, t}=b_{1} M Q M_{i, t}+b_{2} Q J_{N O T i, t}+b_{3} S P 500_{t}+b_{4} V I X_{t}+\varepsilon_{2, t}$

As an instrument for $Q J_{i, t}$ we use $Q J_{N O T i, t}$, which is the daily average QJ activity of other stocks listed on the same exchange, in the same market capitalization grouping (LARGE, MEDIUM, SMALL)(excluding stock i). Similarly, as an instrument for $M Q M_{i, t}$ we use $M Q M_{N O T i, t}$, which is the average time-weighted relative spread for other stocks listed on the same exchange, in the same market capitalization grouping (LARGE, MEDIUM, SMALL)(excluding stock i). We estimate the simultaneous equation model by pooling observations across all stocks and days in the sample. To make the pooling meaningful, we de-mean all variables by deducting the stock-specific average and scale all variables by dividing by the stock-specific standard deviation to control for stock fixed effects. We report the estimated coefficients on top and p-values below. Estimation is done with 2SLS with two-way clustered standard errors (i.e., stock and day).

\begin{tabular}{|c|c|c|c|c|c|c|}
\hline & \multicolumn{3}{|c|}{ Panel A } & \multicolumn{3}{|c|}{ Panel B } \\
\hline & (1) & $(2)$ & (3) & (3) & $(4)$ & (5) \\
\hline & Full sample & Small & Large & Full sample & Small & Large \\
\hline \multirow[t]{2}{*}{$a_{1}$} & $-0.49^{* *}$ & -0.55 & -0.29 & $-0.46^{* *}$ & -0.60 & $-0.27^{*}$ \\
\hline & $(0.007)$ & $(0.070)$ & $(0.164)$ & (0.002) & (0.090) & $(0.046)$ \\
\hline \multirow[t]{2}{*}{$a_{2}$} & $0.63^{* * *}$ & $0.58^{* * *}$ & $0.68^{* * *}$ & $0.61^{* * *}$ & $0.50^{* * * *}$ & $0.67^{* * *}$ \\
\hline & $(0.000)$ & $(0.000)$ & $(0.000)$ & $(0.000)$ & $(0.000)$ & $(0.000)$ \\
\hline \multirow[t]{2}{*}{$a_{3}$} & & & & -0.028 & $-0.068^{*}$ & -0.017 \\
\hline & & & & $(0.057)$ & $(0.012)$ & (0.608) \\
\hline \multirow{2}{*}{$a_{4}$} & & & & 0.00014 & 0.00027 & 0.00017 \\
\hline & & & & $(0.781)$ & $(0.774)$ & $(0.846)$ \\
\hline Observations & 7560 & 2520 & 2520 & 7560 & 2520 & 2520 \\
\hline \multirow{2}{*}{$b_{1}$} & $-0.27^{* * *}$ & $-0.26^{*}$ & $-0.23^{*}$ & $-0.26^{* * *}$ & $-0.32^{* *}$ & $-0.21^{*}$ \\
\hline & $(0.000)$ & $(0.024)$ & $(0.015)$ & $(0.000)$ & (0.008) & (0.011) \\
\hline \multirow[t]{2}{*}{$b_{2}$} & $0.35 * * *$ & $0.28 * *$ & $0.54^{* * *}$ & $0.35^{* * *}$ & $0.26^{*}$ & $0.53^{* * *}$ \\
\hline & $(0.000)$ & $(0.005)$ & $(0.000)$ & $(0.000)$ & $(0.013)$ & $(0.000)$ \\
\hline \multirow[t]{2}{*}{$b_{3}$} & & & & 0.0086 & -0.035 & 0.026 \\
\hline & & & & $(0.525)$ & $(0.230)$ & $(0.291)$ \\
\hline \multirow[t]{2}{*}{$b_{4}$} & & & & -0.000084 & 0.000084 & -0.00013 \\
\hline & & & & $(0.868)$ & $(0.930)$ & $(0.883)$ \\
\hline Observations & 7560 & 2520 & 2520 & 7560 & 2520 & 2520 \\
\hline
\end{tabular}


Table 8: NASDAQ and NYSE mid-crossing and time-weighted relative spread

This table reports the results from the analysis of the relationship between mid-crossing activity and time-weighted relative spread. We measure mid-crossing activity as MID, which is defined as 100 times daily mid-crossing dollar volume on ADF divided by daily consolidated dollar volume (volume computed during trading hours 9:30 AM - 4:00 PM ET). Due to the potential simultaneity between time-weighted relative spread and mid-crossing activity, in Panel A we estimate the following two-equation simultaneous model for MID and the time-weighted relative spread $(\mathrm{MQM})$ :

$M Q M_{i, t}=a_{1} M I D_{i, t}+a_{2} M Q M_{N O T i, t}+\varepsilon_{1, t}$

$Q J_{i, t}=b_{1} M Q M_{i, t}+b_{2} M I D_{N O T i, t}+\varepsilon_{2, t}$

In Panel B, we estimate the following two-equation simultaneous model, including as control, the return on SP500 and the VIX:

$M Q M_{i, t}=a_{1} M I D_{i, t}+a_{2} M Q M_{N O T i, t}+a_{3} S P 500_{t}+a_{4} V I X_{t}+\varepsilon_{1, t}$

$M I D_{i, t}=b_{1} M Q M_{i, t}+b_{2} M I D_{N O T i, t}+b_{3} S P 500_{t}+b_{4} V I X_{t}+\varepsilon_{2, t}$

As an instrument for $M I D_{i, t}$ we use $M I D_{N O T i, t}$, which is the daily average MID activity of other stocks listed on the same exchange, in the same market capitalization grouping (LARGE, MEDIUM, SMALL)(excluding stock i). Similarly, as an instrument for $M Q M_{i, t}$ we use $M Q M_{N O T i, t}$, which is the average time-weighted relative spread for other stocks listed on the same exchange, in the same market capitalization grouping (LARGE, MEDIUM, SMALL)(excluding stock i). We estimate the simultaneous equation model by pooling observations across all stocks and days in the sample. To make the pooling meaningful, we de-mean all variables by deducting the stock-specific average and scale all variables by dividing by the stock-specific standard deviation to control for stock fixed effects. We report the estimated coefficients on top and p-values below. Estimation is done with 2SLS with two-way clustered standard errors (i.e., stock and day).

\begin{tabular}{|c|c|c|c|c|c|c|}
\hline & \multicolumn{3}{|c|}{ Panel A } & \multicolumn{3}{|c|}{ Panel B } \\
\hline & (1) & $(2)$ & (3) & $(4)$ & $(5)$ & (6) \\
\hline & Full sample & Small & Large & Full sample & Small & Large \\
\hline \multirow[t]{2}{*}{$a_{1}$} & -0.32 & -0.076 & -0.58 & -0.30 & -0.13 & -0.54 \\
\hline & $(0.157)$ & $(0.817)$ & $(0.245)$ & (0.103) & $(0.685)$ & (0.199) \\
\hline \multirow[t]{2}{*}{$a_{2}$} & $0.74^{* * *}$ & $0.72^{* * *}$ & $0.67^{* * *}$ & $0.71^{* * *}$ & $0.66^{* * *}$ & $0.66^{* * *}$ \\
\hline & $(0.000)$ & $(0.000)$ & $(0.000)$ & $(0.000)$ & $(0.000)$ & $(0.000)$ \\
\hline \multirow{2}{*}{$a_{3}$} & & & & $-0.039 * * *$ & $-0.057^{*}$ & -0.015 \\
\hline & & & & $(0.000)$ & $(0.020)$ & $(0.624)$ \\
\hline \multirow[t]{2}{*}{$a_{4}$} & & & & 0.00023 & 0.00027 & 0.00020 \\
\hline & & & & $(0.636)$ & $(0.758)$ & $(0.841)$ \\
\hline Observations & 7560 & 2520 & 2520 & 7560 & 2520 & 2520 \\
\hline \multirow[t]{2}{*}{$b_{1}$} & $-0.13^{* *}$ & -0.045 & $-0.22^{*}$ & $-0.12^{* *}$ & -0.067 & $-0.20^{*}$ \\
\hline & $(0.002)$ & $(0.362)$ & $(0.015)$ & (0.003) & $(0.380)$ & $(0.010)$ \\
\hline \multirow{2}{*}{$b_{2}$} & $0.22^{* * *}$ & $0.28^{* * *}$ & $0.20^{*}$ & $0.22^{* * *}$ & $0.27^{* * *}$ & 0.19 \\
\hline & $(0.000)$ & $(0.000)$ & $(0.033)$ & $(0.000)$ & $(0.000)$ & $(0.057)$ \\
\hline \multirow[t]{2}{*}{$b_{3}$} & & & & 0.0040 & -0.016 & 0.027 \\
\hline & & & & $(0.789)$ & $(0.583)$ & $(0.212)$ \\
\hline \multirow[t]{2}{*}{$b_{4}$} & & & & 0.0000055 & 0.00015 & -0.000072 \\
\hline & & & & $(0.992)$ & $(0.875)$ & $(0.933)$ \\
\hline Observations & 7560 & 2520 & 2520 & 7560 & 2520 & 2520 \\
\hline
\end{tabular}




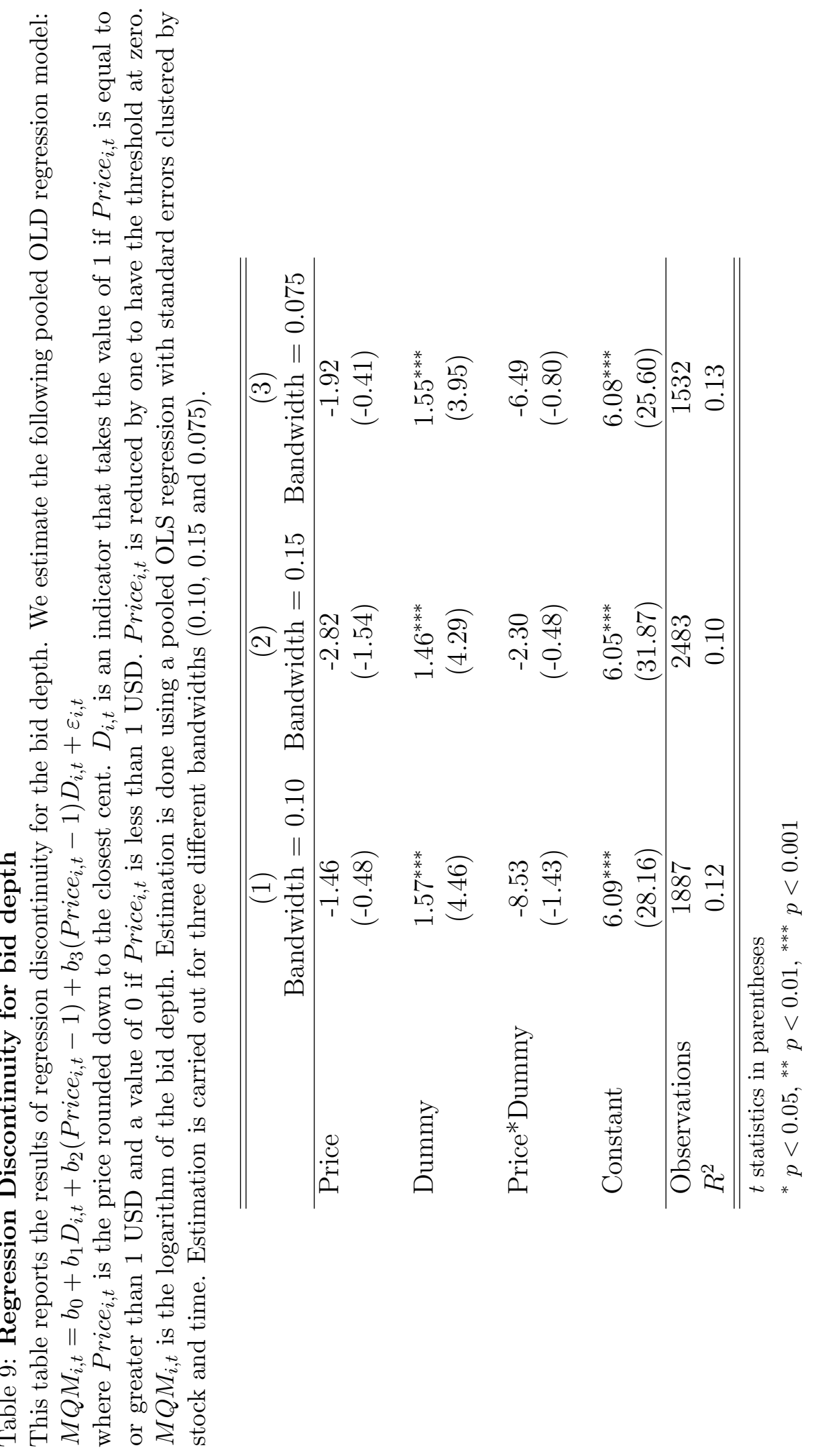




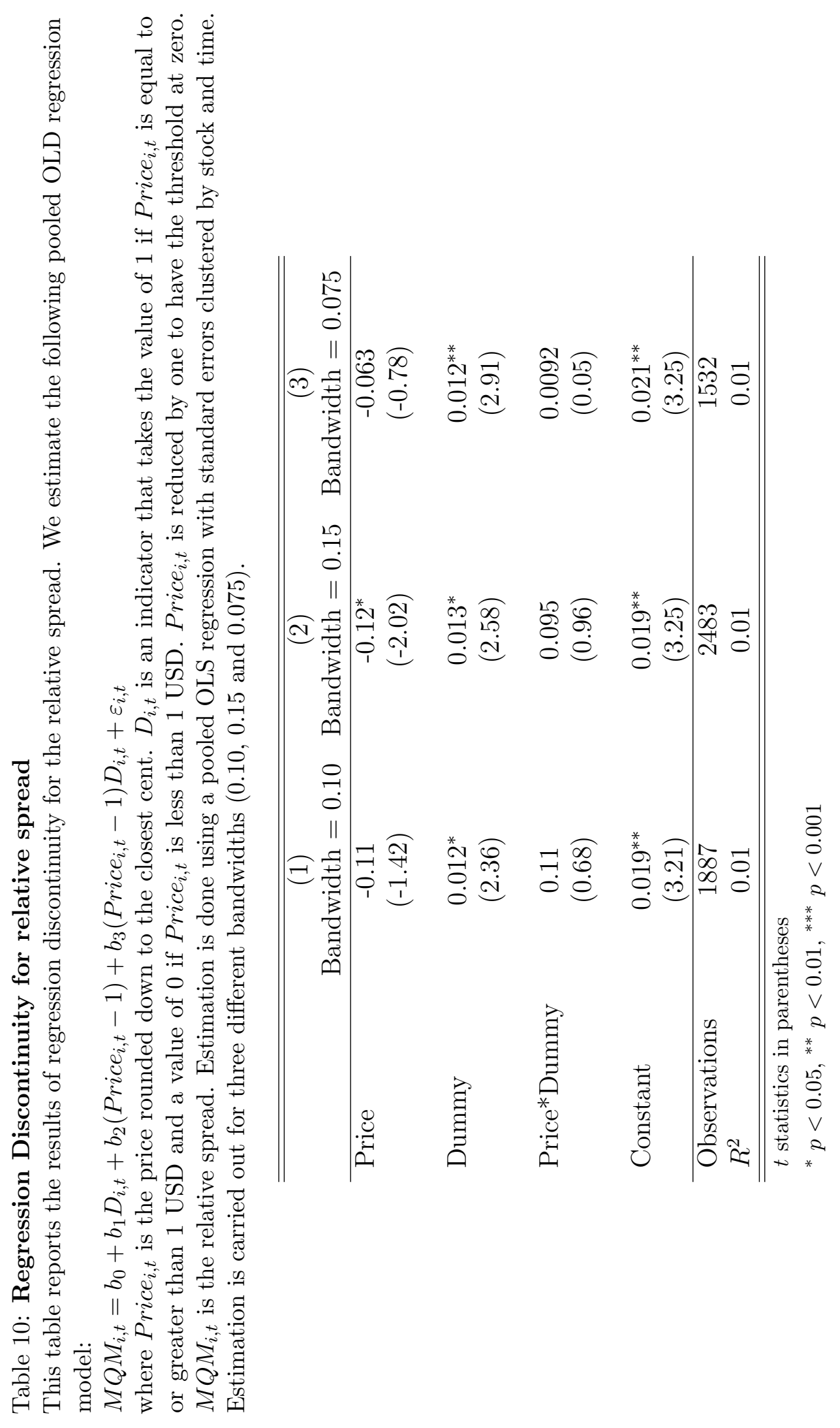




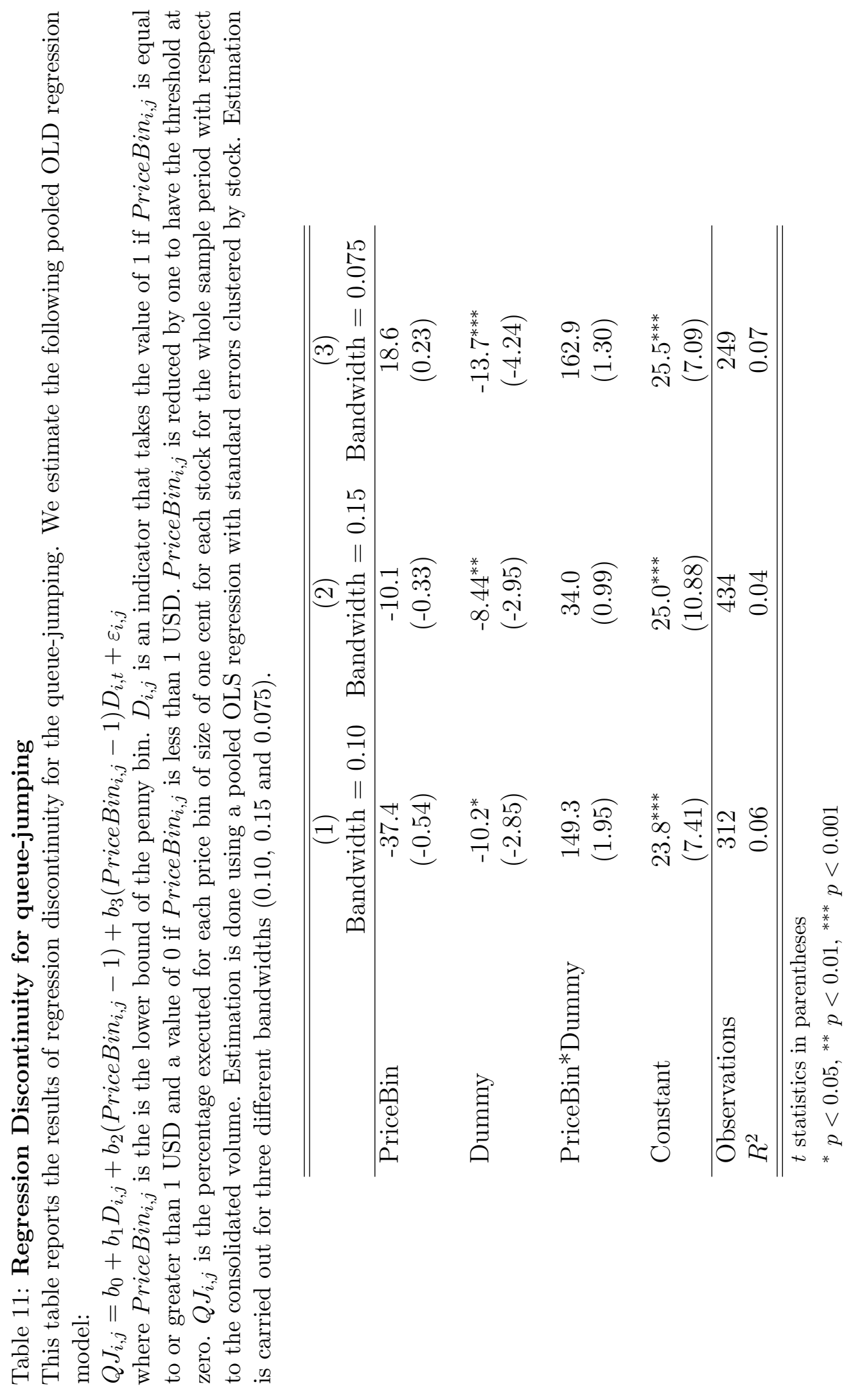

